\title{
Codegree thresholds for covering 3-uniform hypergraphs
}

\author{
Victor Falgas-Ravry \\ Department of Mathematics \\ Vanderbilt University, Nashville \\ Tennessee, U.S.A. \\ victor.falgas-ravry@vanderbilt.edu
}

\author{
Yi Zhao * \\ Department of Mathematics \\ Georgia State University, Atlanta \\ Georgia, U.S.A. \\ yzhao6@gsu.edu
}

\begin{abstract}
Given two 3-uniform hypergraphs $F$ and $G$, we say that $G$ has an $F$-covering if we can cover $V(G)$ by copies of $F$. The minimum codegree of $G$ is the largest integer $d$ such that every pair of vertices from $V(G)$ is contained in at least $d$ triples from $E(G)$. Define $c_{2}(n, F)$ to be the largest minimum codegree among all $n$-vertex 3 -graphs $G$ that contain no $F$-covering. This is a natural problem intermediate (but distinct) from the well-studied Turán problems and tiling problems. In this paper, we determine $c_{2}\left(n, K_{4}\right)$ (for $n>98$ ) and the associated extremal configurations (for $n>998$ ), where $K_{4}$ denotes the complete 3 -graph on 4 vertices. We also obtain bounds on $c_{2}(n, F)$ which are apart by at most 2 in the cases where $F$ is $K_{4}^{-}$( $K_{4}$ with one edge removed), $K_{5}^{-}$, and the tight cycle $C_{5}$ on 5 vertices.
\end{abstract}

\section{Introduction}

\subsection{Notation}

Given a set $A$ and a positive integer $k$, we write $A^{(k)}$ for the collection of $k$-element subsets of $A$. We use $[n]$ as a shorthand for the collection of the first $n$ natural numbers, $[n]=\{1,2, \ldots, n\}$. We shall often consider pairs or triples of vertices; when there is no risk of confusion, we write $a b$ and $a b c$ as a shorthand for $\{a, b\}$ and $\{a, b, c\}$ respectively. A $k$-uniform hypergraph, or $k$-graph, is a pair $G=(V, E)$, where $V$ is a set of vertices, and $E \subseteq V^{(k)}$ is a collection of $k$-subsets of $V$, which form the edges of $G$. A subgraph of $G$ is a $k$-graph $H$ with $V(H) \subseteq V(G)$ and $E(H) \subseteq E(G)$. The degree of a vertex $x \in V(G)$, which we denote by $d(x)$, is the number of edges of $G$ containing $x$. The minimum degree $\delta_{1}(G)$ of $G$ is the minimum of $d(x)$ over all vertices $x \in V(G)$.

In this paper, we will focus on 3-graphs $G=(V, E)$ and another degree-like quantity, and its minimum: the codegree of a pair $x y \in V^{(2)}$, denoted by $d(x, y)$, is the number of edges of $G$ containing the pair $x y$. We write $\Gamma(y, z)$ for the neighbourhood of the pair $x y$, i.e. the set of $z \in V \backslash\{x, y\}$ such that $x y z \in E(G)$. The minimum codegree of $G$ is $\delta_{2}(G)=\min _{x y \in V^{(2)}} d(x, y)$. The link graph of a vertex $x \in V(G)$ is the collection $G_{x}$ of all pairs $u v$ such that $x u v \in E(G)$. The degree of $u$ in $G_{x}$ is the number of vertices $v$ such that $u v \in G_{x}$; note this is exactly the codegree of $x$ and $u$. Finally we define the edit distance between two 3-graphs $G$ and $G^{\prime}$ on the same vertex set to be the minimum number of changes required to make $G$ isomorphic to $G^{\prime}$, where a change consists in replacing an edge by a non-edge and vice-versa.

\footnotetext{
${ }^{*}$ Research partially supported by NSF Grant DMS-1400073.
} 


\subsection{The problem}

Let $F$ be a fixed 3-graph on $t$ vertices with at least one 3-edge. A 3 -graph $G$ has an $F$-covering if we can cover $V(G)$ with $F$-subgraphs (subgraphs that are isomorphic to $F$ ). For $n \geq t$ and $i=1,2$, we define

$$
c_{i}(n, F)=\max \left\{\delta_{i}(G):|V(G)|=n \text { and } G \text { does not have an } F \text {-covering }\right\} .
$$

and call $c_{1}(n, F)$ the covering degree-threshold and $c_{2}(n, F)$ the covering codegree-threshold of $F$.

The covering threshold $c_{i}(n, F)$ was introduced by Han, Zang and Zhao [14] when they studied the minimum degree that guarantees the existence of a $K$-tiling, where $K$ is a complete 3 -partite 3-graph. It was shown implicitly in [14] that $c_{1}(n, K)=(6-4 \sqrt{2}+o(1))\left(\begin{array}{c}n \\ 2\end{array}\right)$ if $K$ has at least two vertices in each part (in contrast, it is easy to see that $c_{1}(n, K)=o\left(n^{2}\right)$ if some part of $K$ has only one vertex). It was also noted that $c_{1}(n, F)=(1-1 /(\chi(F)-1)+o(1)) n$ for all graphs $F$, where $\chi(F)$ is the chromatic number of $F$.

Our objective in this paper is to study the behaviour of the function $c_{2}(n, F)$ for various 3-graphs $F$. In other words, we seek to determine what codegree condition is necessary to guarantee that all vertices in a 3-graph $G$ are contained in copies of $F$. When determining the exact value of $c_{2}(F, n)$ is difficult, we may ask instead for its asymptotic behaviour. It can be shown (see Section ??) that the limit

$$
c_{2}(F)=\lim _{n \rightarrow \infty} \frac{c_{2}(n, F)}{n-2}
$$

exists 1 We call $c_{2}(F)$ the covering codegree-density of $F$.

Let us introduce the 3 -graphs relevant to the present work. Let $K_{t}=\left([t],[t]^{(3)}\right)$ denote the complete 3 -graph on $t$ vertices, and let $K_{t}^{-}$denote the 3 -graph obtained from $K_{t}$ by removing one 3 -edge. The strong or tight $t$-cycle is the 3 -graph $C_{t}$ on $[t]$ with 3 -edges $\{123,234,345, \ldots,(t-$ $2)(t-1) t,(t-1) t 1, t 12\}$. We denote by $F_{3,2}$ the 3 -graph $([5],\{123,124,125,345\})$. Finally a Steiner Triple System (STS) is a 3-graph in which every pair of vertices is contained in exactly one 3-edge; it is a 168 years old result of Kirkman [19] that a STS on $t$ vertices exists if and only if $t \equiv 1,3$ mod 6. The Fano plane is the unique (up to isomorphism) STS on 7 vertices, which we denote by Fano.

\subsection{Motivation and related work in extremal hypergraph theory}

Before we state our results, let us give some motivation and background for our problem. Let $F$ be a fixed 3-graph on $t$ vertices with at least one 3-edge. A 3-graph $G$ is $F$-free if it does not contain a copy of $F$ as a subgraph. Further $G$ has an $F$-tiling, or $F$-factor, if we can cover $V(G)$ with vertexdisjoint $F$-subgraphs. There has been much research into the degree and/or codegree conditions needed ensure the existence of an $F$-subgraph or of an $F$-factor in a 3-graph $G$. Determining the degree/codegree condition necessary to guarantee an $F$-covering is intermediate between these two well-studied problems. As we show in the next subsection, the existence, covering, and tiling problems give rise to different thresholds in their codegree versions, so that our work is novel. It is hoped that studying the properties of the covering codegree threshold function $c_{2}(n, F)-$ such as

\footnotetext{
${ }^{1}$ This is a direct corollary of the proof of Proposition 6 from [7] on the existence of conditional codegree density (with an uncovered vertex $x$ used as the conditional subgraph $H$ ), or can be proved in the same way as the existence of the usual codegree density $\gamma(F)$ in [27].
} 
supersaturation, discussed in Section 4, which could be useful for applying semi-random methods to tiling problems - will lead to insights about both the existence and tiling problems.

The Turán number ex $(n, F)$ of $F$ is the maximum number of 3-edges an $F$-free 3-graph on $n$ vertices can have. The codegree threshold $\operatorname{ex}_{2}(n, F)$ of $F$ is the maximum of $\delta_{2}(G)$ over all $F$-free 3-graphs $G$ on $n$ vertices. It is well-known that $\operatorname{ex}(n, F) /\left(\begin{array}{l}n \\ 3\end{array}\right)$ tends to a limit $\pi(F)$ as $n \rightarrow \infty$; this limit is known as the Turán density of $F$. Similarly, $\operatorname{ex}_{2}(n, F) /(n-2)$ tends to a limit $\gamma(F)$ called the codegree density or 2-Turán density of $F$ as $n \rightarrow \infty$. The extremal theory of 3-graphs and within it the study of Turán-type problems have received extensive attention from the combinatorics community since the 1950s, with strenuous efforts devoted in particular to the (in)famous and still-open conjecture of Turán [32] that $\pi\left(K_{4}\right)=5 / 9$. See the surveys of Füredi [10] and Keevash [16] for an overview of results. There has been significant interest in other extremal quantities, and in particular in codegree densities for 3-graphs. The first result on codegree density was due to Mubayi [25], who showed $\gamma($ Fano $)=\frac{1}{2}$. Keevash and Zhao [18] determined the codegree densities of some projective geometries, which included the Fano plane as a special case. The codegree threshold for the Fano plane was determined by Keevash [15] via hypergraph regularity and later by DeBiasio and Jiang [5] by direct combinatorial means. Mubayi and Zhao [27] studied general properties of codegree density, while Falgas-Ravry [6] gave examples of non-isomorphic lower bound constructions for $\gamma\left(K_{t}\right)$. More recently Falgas-Ravry, Marchant, Pikhurko and Vaughan [7] determined the codegree threshold of $F_{3,2}$, and Falgas-Ravry, Pikhurko and Vaughan [8] showed $\gamma\left(K_{4}^{-}\right)=\frac{1}{4}$ via a flag algebra computation, resolving a conjecture of Czygrinow and Nagle [29]. Another conjecture of Czygrinow and Nagle [4] remains open, namely that $\gamma\left(K_{4}\right)=\frac{1}{2}$. Certainly $\gamma(F) \leq c_{2}(F)$ for any 3 -graph $F$, and it may be hoped that giving good upper bounds for the latter may also help bounding the former.

In addition to these Turán-type problems, there has been much research activity on the problem of determining thresholds for the existence of $F$-factors. The situation for ordinary (2-)graphs is now well-understood: the celebrated Hajnal-Szemerédi theorem [12] gives the exact minimum degree condition guaranteeing the existence of $F$-factors in an $n$-vertex graph when $F$ is a clique, while Kühn and Osthus [21] determined the minimum degree condition for general graphs $F$ up to an additive constant. On the other hand, until recently not much was known about tiling for $k$-graphs when $k \geq 3$. While there has been a spate of results in the last few years, see [2, 3, 11, 13, 17, 20, 22, 23, 28, 31], many more open problems remain. We refer to the surveys of Rödl and Ruciński 30] and Zhao [33] for a more detailed discussion of the area, and briefly mention below four results relevant to the present work. For $i \in\{1,2\}$ and $n \equiv 0 \bmod |V(F)|$, let

$$
t_{i}(n, F)=\max \left\{\delta_{i}(G):|V(G)|=n \text { and } G \text { does not have an } F \text {-factor }\right\} .
$$

Trivially $c_{i}(n, F) \leq t_{i}(n, F)$ for any 3-graph $F$ with at least one edge. Lo and Markström [23, 22] determined $t_{2}(n, F)$ asymptotically when $F=K_{4}$ and $F=K_{4}^{-}$. Independently Keevash and Mycroft [17] determined $t_{2}\left(n, K_{4}\right)$ exactly, and recently Han, Lo, Treglown and Zhao [13] determined $t_{2}\left(n, K_{4}^{-}\right)$exactly as well, in both cases for $n$ sufficiently large. Finally in [14] Han, Zang and Zhao asymptotically determined $t_{1}(n, K)$ for all complete 3-partite 3-graphs $K$. In particular, they showed that $t_{1}(n, K)=c_{1}(n, K)=(6-4 \sqrt{2}+o(1))\left(\begin{array}{l}n \\ 2\end{array}\right)$ for certain $K$. This gives further motivation for the present paper: by determining $c_{2}(n, F)$ for 3 -graphs $F$, we may hope likewise to shed light on $t_{2}(n, F)$ and facilitate its (asymptotic) computation. 


\subsection{Results}

In this paper, we determine the codegree covering threshold for $K_{4}$ for sufficiently large $n$.

Theorem 1.1. For every $n \in \mathbb{N},\left\lfloor\frac{2 n-5}{3}\right\rfloor \leq c_{2}\left(K_{4}, n\right) \leq\left\lfloor\frac{2 n-3}{3}\right\rfloor$. Furthermore, for every $n>98$,

$$
c_{2}\left(n, K_{4}\right)=\left\lfloor\frac{2 n-5}{3}\right\rfloor \text {. }
$$

In addition, we determine $c_{2}(F)$ when $F$ is $K_{4}^{-}$, the strong 5 -cycle $C_{5}$, and $K_{5}^{-}$- in fact in each case we give upper and lower bounds on $c_{2}(n, F)$ differing by at most 2 .

Theorem 1.2. Suppose $n=6 m+r$ for some $r \in\{0,1,2,3,4,5\}$ and $m \in \mathbb{N}$, with $n \geq 7$. Then

$$
c_{2}\left(n, K_{4}^{-}\right)= \begin{cases}2 m-1 \text { or } 2 m & \text { if } r=0 \\ 2 m & \text { if } r \in\{1,2\} \\ 2 m \text { or } 2 m+1 & \text { if } r \in\{3,4\} \\ 2 m+1 & \text { if } r=5 .\end{cases}
$$

In particular, $c_{2}\left(K_{4}^{-}\right)=\frac{1}{3}$.

Theorem 1.3. $\left\lfloor\frac{n-3}{2}\right\rfloor \leq c_{2}\left(n, C_{5}\right) \leq\left\lfloor\frac{n}{2}\right\rfloor$. In particular, $c_{2}\left(C_{5}\right)=\frac{1}{2}$.

Interestingly, there is no unique stable near-extremal configuration for Theorem 1.3, at least two configurations at edit distance $\Omega\left(n^{3}\right)$ of each other exist, see Remark 3.4.

Theorem 1.4. $\left\lfloor\frac{2 n-5}{3}\right\rfloor \leq c_{2}\left(n, K_{5}^{-}\right) \leq\left\lfloor\frac{2 n-2}{3}\right\rfloor$. In particular, $c_{2}\left(K_{5}^{-}\right)=\frac{2}{3}$.

Let us compare the Turán density $\pi$, the (existence) codegree density $\gamma$, the covering codegree density $c_{2}$, and the tiling codegree density $t_{2}$ of $K_{4}, K_{4}^{-}$, and $C_{5}$ in the following table (for a 3-graph $F$ of order $f$, define $t_{2}(F)=\lim _{n=m f \rightarrow \infty} t_{2}(n, F) /(n-2)$ if this limit exists). In the table question marks indicate conjectures, except for $t_{2}\left(C_{5}\right)$, for which we are not aware of any conjecture.

\begin{tabular}{|c|c|c|c|c|}
\hline & $\gamma$ & $\pi$ & $c_{2}$ & $t_{2}$ \\
\hline$K_{4}$ & $\frac{1}{2} ?[4]$ & $\frac{5}{9} ?[32]$ & $\frac{2}{3}$ & $\frac{3}{4}[17,23]$ \\
\hline$K_{4}^{-}$ & $\frac{1}{4}[8]$ & $\frac{2}{7} ?[9]$ & $\frac{1}{3}$ & $\frac{1}{2}[22]$ \\
\hline$C_{5}$ & $\frac{1}{3} ?[24]$ & $2 \sqrt{3}-3 ?[26]$ & $\frac{1}{2}$ & $?$ \\
\hline
\end{tabular}

Finally we give bounds on $c_{2}$ (Fano), $c_{2}\left(F_{3,2}\right)$ and $c_{2}\left(K_{t}\right)$ for $t \geq 5$, and pose a number of questions.

Our paper is structured as follows. In Section 2, we determine the codegree covering threshold for $K_{4}$ and characterize the extremal configurations. In Section 3 , we prove our bounds on $c_{2}(n, F)$ for the other 3-graphs $F$ mentioned above. We end in Section 4 with some discussion and questions.

\section{The covering codegree threshold for $K_{4}$}

In this section we determine the codegree threshold $c_{2}\left(n, K_{4}\right)$. We give a lower bound construction in Section 2.1 and prove the upper bound in Section 2.2. Finally, in Section 2.3 we provide other extremal constructions, and state a stability theorem that helps to show that these constructions are all possible extremal configurations; as the proofs of these latter results are similar to the proof of Theorem 1.1 we defer them to the appendix. 


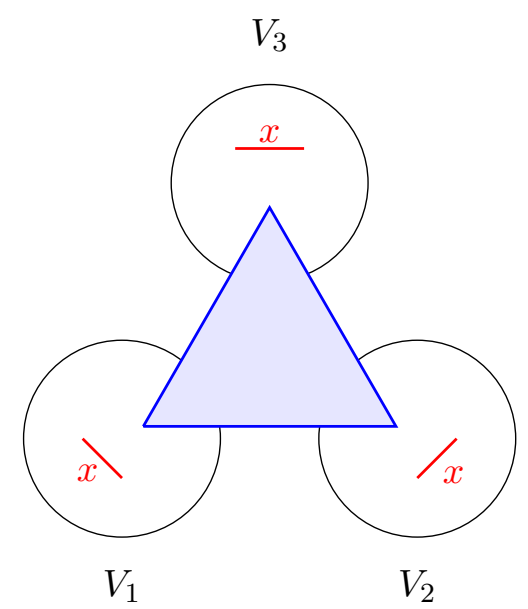

Figure 1: The complement of $F_{1}(n)$. The red pairs and the blue triples are absent from the link graph of $x$ in $F_{1}$ and from $E\left(F_{1}\right)$ respectively.

\section{$2.1 \quad$ Lower bound}

Proof of the lower bound in Theorem [1.1. We construct a 3-graph $F_{1}(n)$ on $V=[n]$. Select a special vertex $x$. Split the remainder of the vertices into three parts $V_{1} \sqcup V_{2} \sqcup V_{3}=V \backslash\{x\}$ with sizes as equal as possible,

$$
\left|V_{3}\right|-1 \leq\left|V_{1}\right| \leq\left|V_{2}\right| \leq\left|V_{3}\right| .
$$

Put in as the link graph of $x$ all pairs between distinct parts, i.e. add in all triples of the form $x V_{i} V_{j}$ for $i \neq j$. Further, add in all triples not containing $x$ and meeting at most two of the three parts $\left(V_{i}\right)_{i=1}^{3}$. Denote the resulting 3 -graph by $F_{1}=F_{1}(n)$. The complement of $F_{1}(n)$ is shown in Figure 1.

Observe that $x$ is contained in no copy of $K_{4}$ in $F_{1}$ : the only triangles in the link graph of $x$ are tripartite, and thus are not covered by any triple of the 3-graph. Let us now compute the minimum codegree of $F_{1}$. For $v_{i}, v_{i}^{\prime} \in V_{i}$ and $v_{i+1} \in V_{i+1}$, we have $d\left(v_{i}, x\right)=n-1-\left|V_{i}\right|, d\left(v_{i}, v_{i}^{\prime}\right)=n-3$ and $d\left(v_{i}, v_{i+1}\right)=n-2-\left|V_{i+2}\right|$. The minimum codegree $\delta_{2}\left(F_{1}\right)$ is thus $n-2-\left\lceil\frac{n-1}{3}\right\rceil$, attained by pairs $\left(v_{1}, v_{2}\right) \in V_{1} \times V_{2}$. Writing $n=3 m+r$ with $r \in\{0,1,2\}$ and $m \in \mathbb{N}$, we have shown that

$$
c_{2}\left(3 m+r, K_{4}\right) \geq \delta_{2}\left(F_{1}\right)= \begin{cases}2 m-2 & \text { if } r=0 \\ 2 m-1 & \text { if } r=1 \text { or } 2 .\end{cases}
$$

This lower bound can be expressed more compactly as $c_{2}\left(n, K_{4}\right) \geq\left\lfloor\frac{2 n-5}{3}\right\rfloor$.

\subsection{Upper bound}

Let us give a general upper bound for $c_{2}(n, F)$, which turns out to be surprisingly close to the truth in the case of $F=K_{4}$.

Lemma 2.1. Given a 3-graph $F$ with at least one 3 -edge, let $r$ be the maximum of $\delta_{1}\left(F^{\prime}\right)$ among all subgraphs $F^{\prime}$ of $F$. Then $c_{2}(n, F) \leq\lfloor(1-1 / r) n+(|V(F)|-2 r-1) / r\rfloor$. 
Proof. Assume that $F$ contains $f$ vertices. We order the vertices of $F$ as $x_{1}, \ldots, x_{f}$ such that $x_{i}$ is a vertex of minimum degree in the subgraph $F-\left\{x_{i+1}, \ldots, x_{f}\right\}$. As $r=\max \delta_{1}\left(F^{\prime}\right)$ among all subgraphs $F^{\prime}$ of $F$, we know that $x_{i}$ has at most $r$ neighbours among $x_{1}, \ldots, x_{i}$.

Let $G$ be a 3 -graph on $n$ vertices such that

$$
\delta_{2}:=\delta_{2}(G)>\frac{r-1}{r} n+\frac{f-1}{r}-2 .
$$

Fix a vertex $v_{1}$ of $G$. We will find a copy of $F$ in $G$ by first mapping $x_{1}$ to $v_{1}, x_{2}$ to any other vertex $v_{2}$, and $x_{3}$ to any $v_{3} \in \Gamma_{G}\left(v_{1}, v_{2}\right)$. Suppose that $x_{1}, \ldots, x_{i}$ have been embedded to $v_{1}, \ldots, v_{i}$. In order to embed $x_{i+1}$, we consider the neighbours of $x_{i+1}$ among $x_{1}, \ldots, x_{i}$. There are $t \leq r$ such neighbours and they are mapped to pairs $p_{1}, \ldots p_{t}$ of $v_{1}, \ldots, v_{i}$. Each $p_{j}$ has at least $\delta_{2}$ neighbours in $G$ and thus at most $n-2-\delta_{2}$ non-neighbours in $V(G) \backslash\left\{v_{1}, \ldots, v_{i}\right\}$. By the definition of $\delta_{2}$ and $i \leq f-1$, we have $r\left(n-2-\delta_{2}\right)<n-i$. Hence there exists a vertex $v_{i+1} \in V(G) \backslash\left\{v_{1}, \ldots, v_{i}\right\}$ such that $v_{i+1}$ is a common neighbour of $p_{1}, \ldots p_{t}$. Continuing this process, we obtain a copy of $F$ as desired.

Remark 2.2. The proof of Lemma[2.1 actually shows that if $\delta_{2}(G)>(1-1 / r) n+(|V(F)|-2 r-1) / r$ then every triple of $E(G)$ is covered by an F-subgraph.

Applying Lemma 2.1 with $F=K_{4}$ and $r=3$, we obtain that $c_{2}\left(n, K_{4}\right) \leq\left\lfloor\frac{2 n-3}{3}\right\rfloor$. When $n \equiv 1$ mod 3, this implies that $c_{2}\left(n, K_{4}\right) \leq\left\lfloor\frac{2 n-5}{3}\right\rfloor$. Together with the lower bound $c_{2}\left(n, K_{4}\right) \geq\left\lfloor\frac{2 n-5}{3}\right\rfloor$, we obtain $c_{2}\left(n, K_{4}\right)=\left\lfloor\frac{2 n-5}{3}\right\rfloor$ immediately.

When $n \equiv 0$ or $2 \bmod 3$, more work is required to reduce the upper bound to $\left\lfloor\frac{2 n-5}{3}\right\rfloor$. In both cases, we shall make use of the following simple observation.

Lemma 2.3. Let $G$ be a 3-graph on $n \geq 4$ vertices. Suppose that $x \in V(G)$ is not covered by any copy of $K_{4}$ and there exists $a, b, c \in V(G)$ such that $a b x, b c x, a c x \in E(G)$ (thus abc $\notin E(G)$ ). Let $S=\{a, b, c, x\}$ and for each vertex $y \in V(G) \backslash S$, let $S_{y}$ consist of all the pairs of $S$ that make a 3 -edge with $y$ in $G$. Then $S_{y}$ must be a subset of one of the following sets:

$$
\begin{gathered}
S^{1, c}=\{a x, b x, a c, b c\}, \quad S^{1, b}=\{a x, c x, a b, b c\}, \quad S^{1, a}=\{b x, c x, a b, a c\}, \\
S^{2, a}=\{a b, a c, b c, a x\}, S^{2, b}=\{a b, a c, b c, b x\}, S^{2, c}=\{a b, a c, b c, c x\}, S^{3}=\{a x, b x, c x\} .
\end{gathered}
$$

In particular, $\left|S_{y}\right| \leq 4$.

Proof of $c_{2}\left(n, K_{4}\right) \leq\lfloor(2 n-5) / 3\rfloor$ when 3 divides $n$. Suppose $n=3 m$ for some integer $m \geq 2$ (so that $\lfloor(2 n-5) / 3\rfloor=2 n / 3-2)$. Let $G=(V, E)$ be a 3 -graph on $n$ vertices with $\delta_{2}(G) \geq 2 n / 3-1$. We claim that all vertices of $G$ are covered by copies of $K_{4}$. Suppose instead, that some vertex $x \in V$ is not contained in a copy of $K_{4}$. Since the minimum degree in the link graph $G_{x}$ of $x$ is at least $2 n / 3-1>(n-1) / 2$, there exists a triangle $\{a b, b c, a c\}$ in $G_{x}$. This implies that $a b c \notin E$. Set $S=\{a, b, c, x\}$. For each vertex $y \in V \backslash S$, by Lemma 2.3, at most four pairs of $S$ form edges of $G$ with $y$. Thus, by the codegree assumption,

$$
6\left(\frac{2 n}{3}-1\right) \leq d(a, x)+d(b, x)+d(c, x)+d(a, b)+d(b, c)+d(c, a) \leq 4(n-4)+9,
$$

a contradiction. 
When $n \equiv 2 \bmod 3$, we start the proof in the same way. However, since we only have $\delta_{2}(G) \geq$ $(2 n-4) / 3$, we will not obtain a contradiction until we prove that $G$ has a similar structure as the 3 -graph $F_{1}(n)$ given in Section 2.1,

Proof of $c_{2}\left(n, K_{4}\right) \leq\left\lfloor\frac{2 n-5}{3}\right\rfloor$ when $n \equiv 2 \bmod 3$. Suppose $n=3 m+2>98$. In order to show that $c_{2}\left(n, K_{4}\right) \leq\left\lfloor\frac{2 n-5}{3}\right\rfloor=(2 n-7) / 3$, consider a 3-graph $G=(V, E)$ on $n$ vertices satisfying $\delta_{2}(G) \geq(2 n-4) / 3$.

Suppose that a vertex $x$ of $G$ is not contained in any copy of $K_{4}$. As $(2 n-4) / 3>(n-1) / 2$, the link graph $G_{x}$ contains a triangle $\{a b, b c, a c\}$. Set $S=\{a, b, c, x\}$ and for each $y \in V \backslash S$, define $S_{y}$ as in Lemma 2.3. By Lemma [2.3, $S_{y}$ is a subset of $S^{1, c}, S^{1, b}, S^{1, a}, S^{2, a}, S^{2, b}, S^{2, c}$ or $S^{3}$. For $i \in\{1,2\}$ and $j \in\{a, b, c\}$, write $s_{i, j}$ for the number of vertices $y \in V \backslash S$ for which $S_{y}=S^{i, j}$, and write $s_{i}$ for the sum $s_{i, a}+s_{i, b}+s_{i, c}$. Finally let $s_{0}$ be the number of vertices $y \in V \backslash S$ such that $S_{y} \neq S^{i, j}$ for any $i \in\{1,2\}$ and $j \in\{a, b, c\}$. Note that $\left|S_{y}\right| \leq 3$ for such $y$. We know that $s_{1}+s_{2}+s_{0}=n-4$. Furthermore, by the codegree assumption,

$$
\begin{gathered}
3 \frac{2 n-4}{3} \leq d(a, x)+d(b, x)+d(c, x) \leq 2 s_{1}+s_{2}+3 s_{0}+6, \\
6 \frac{2 n-4}{3} \leq d(a, x)+d(b, x)+d(c, x)+d(a, b)+d(b, c)+d(c, a) \leq 4 s_{1}+4 s_{2}+3 s_{0}+9,
\end{gathered}
$$

Substituting $s_{0}=n-4-s_{1}-s_{2}$ into (11) and (2) yields that $s_{1}+2 s_{2} \leq n-2$ and $s_{1}+s_{2} \geq n-5$, respectively. Combining the two inequalities we have just obtained, we get

$$
s_{2} \leq 3 \quad \text { and } \quad s_{1} \geq n-8 .
$$

We now show that the weight of $s_{1}$ splits almost equally between $s_{1, a}, s_{1, b}, s_{1, c}$. Note that

$$
\frac{2 n-4}{3} \leq d(b, c) \leq n-3-s_{1, a},
$$

from which it follows that $s_{1, a} \leq \frac{n-5}{3}$. Similarly we derive that $s_{1, b}, s_{1, c} \leq(n-5) / 3$. Consequently

$$
s_{1, a}=s_{1}-s_{1, b}-s_{1, c} \geq n-8-2 \frac{n-5}{3}=\frac{n-14}{3} .
$$

Similarly $s_{1, b}$ and $s_{1, c}$ satisfy the same lower bound. Let $A=\left\{y \in V \backslash S: S_{y}=S^{1, a}\right\} \cup\{a\}$, $B=\left\{y \in V \backslash S: S_{y}=S^{1, b}\right\} \cup\{b\}$ and $C=\left\{y \in V \backslash S: S_{y}=S^{1, c}\right\} \cup\{c\}$. Set $V^{\prime}=A \cup B \cup C \cup\{x\}$. Then we have just shown the following lemma.

\section{Lemma 2.4.}

$$
\left|V^{\prime}\right|=1+|A|+|B|+|C| \geq n-4, \quad \text { and } \quad \frac{n-11}{3} \leq|A|,|B|,|C| \leq \frac{n-2}{3} .
$$

Let $\mathcal{B}$ be the collection of 3 -edges of $G$ of the form $x A A, x B B, x C C$ (the 'bad' triples). Let $\mathcal{M}$ be the collection of non-edges of $G$ of the form $x A B, x A C, x B C$ (the 'missing' triples). Viewing $\mathcal{B}$ and $\mathcal{M}$ as 3-graphs on $V^{\prime}$, for two distinct vertices $v_{1}, v_{2} \in V^{\prime}$, we let $d_{\mathcal{B}}\left(v_{1}, v_{2}\right)$ denote their codegree in $\mathcal{B}$ and $d_{\mathcal{M}}\left(v_{1}, v_{2}\right)$ their codegree in $\mathcal{M}$.

Claim 2.5. For every $v \in V^{\prime} \backslash\{x\}, d_{\mathcal{B}}(v, x) \leq 4$. 
Proof. Suppose without loss of generality that $v \in A$. If $v=a$, then $d_{\mathcal{B}}(v, x)=0$ because $G$ contains no 3 -edges of the form $x a A$. We thus assume that $v \neq a$. The bad triples for the pair $(v, x)$ are triples of the form $a^{\prime} v x$ for $a^{\prime} \in A \backslash\{a, v\}$. Suppose $a^{\prime} v x \in \mathcal{B}$. Then since there is no $K_{4}$ in $G$ containing $x$, and since, by the definition of $A, a^{\prime} b x, v b x, a^{\prime} c x$ and $v c x$ are all in $G$, it must be the case that both of $a^{\prime} v b$ and $a^{\prime} v c$ are missing from $G$. Further if $c^{\prime} \in C \cap \Gamma(v, x)$ then all of $c^{\prime} v x, b v x, c^{\prime} b x$ are in $G$, whence $b c^{\prime} v$ is absent from $G$. Similarly for any $b^{\prime} \in B$, at most one of $b^{\prime} c v$, $b^{\prime} x v$ is in $G$. Finally since $b c v \notin E(G), b$ and $c$ are contained in exactly one of $\Gamma(b, v), \Gamma(c, v)$, and $\Gamma(x, v)$. To summarize, a vertex $y$ in $V^{\prime}$ can lie in at most two of $\Gamma(b, v), \Gamma(c, v)$ and $\Gamma(x, v)$ unless $y$ is in $\Gamma_{\mathcal{B}}(x, v)$ (and lies in exactly one of those joint neighbourhoods) or is in $\{b, c, v\}$ (and lies in at most one of those joint neighbourhoods). Together with our codegree assumption, this gives us

$$
\begin{aligned}
3 \frac{2 n-4}{3} \leq d(b, v)+d(c, v)+d(x, v) & \leq 2\left|V^{\prime}\right|-d_{\mathcal{B}}(v, x)-4+3\left(n-\left|V^{\prime}\right|\right) \\
& =3 n-\left|V^{\prime}\right|-4-d_{\mathcal{B}}(v, x) \leq 2 n-d_{\mathcal{B}}(v, x),
\end{aligned}
$$

where we apply $\left|V^{\prime}\right| \geq n-4$ from Lemma 2.4 in the last inequality. It follows that $d_{\mathcal{B}}(v, x) \leq 4$, as claimed.

Claim 2.6. For every $v \in V^{\prime} \backslash\{x\}, d_{\mathcal{M}}(v, x) \leq 8$.

Proof. Suppose without loss of generality that $v \in A$. Then by the codegree assumption, Claim 2.5 and the bound on $|A|$ from Lemma 2.4 we have

$$
\frac{2 n-4}{3} \leq d(v, x) \leq n-1-|A|+d_{\mathcal{B}}(v, x)-d_{\mathcal{M}}(v, x) \leq n-1-\frac{n-11}{3}+4-d_{\mathcal{M}}(v, x),
$$

which gives that $d_{\mathcal{M}}(v, x) \leq 8$ as claimed.

Claim 2.7. For every $y \in V(G) \backslash\{x\}, \Gamma(y, x)$ has a non-empty intersection with at most two of the parts $A, B$ and $C$.

Proof. Let $y \in V(G) \backslash\{x\}$. Set $A_{y}=A \cap \Gamma(x, y), B_{y}=B \cap \Gamma(x, y)$ and $C_{y}=C \cap \Gamma(x, y)$. Suppose none of $A_{y}, B_{y}, C_{y}$ is empty. Fix $a^{\prime} \in A_{y}$. For $b^{\prime} \in B_{y}$, if $b^{\prime} \in \Gamma\left(a^{\prime}, x\right)$, then $a^{\prime} b^{\prime} y \notin E(G)-$ otherwise $\left\{a^{\prime}, b^{\prime}, x, y\right\}$ spans a copy of $K_{4}$. Similarly, for $c^{\prime} \in C_{y} \cap \Gamma\left(a^{\prime}, x\right)$, we have $a^{\prime} c^{\prime} y \notin E(G)$. Hence,

$$
\frac{2 n-4}{3} \leq d\left(a^{\prime}, y\right) \leq n-2-\left|B_{y} \cap \Gamma\left(a^{\prime}, x\right)\right|-\left|C_{y} \cap \Gamma\left(a^{\prime}, x\right)\right| .
$$

Claim 2.6 gives that $d_{\mathcal{M}}\left(a^{\prime}, x\right) \leq 8$. Consequently,

$$
\left|B_{y} \cap \Gamma\left(a^{\prime}, x\right)\right|+\left|C_{y} \cap \Gamma\left(a^{\prime}, x\right)\right|=\left|B_{y}\right|+\left|C_{y}\right|-d_{\mathcal{M}}\left(a^{\prime}, x\right) \geq\left|B_{y}\right|+\left|C_{y}\right|-8
$$

This implies that

$$
\frac{2 n-4}{3} \leq n-2-\left|B_{y}\right|-\left|C_{y}\right|+8,
$$

which yields $\left|B_{y}\right|+\left|C_{y}\right| \leq(n+22) / 3$. Similarly by considering any vertex $b^{\prime} \in B_{y}$ and any vertex $c^{\prime} \in C_{y}$ we obtain that

$$
\left|A_{y}\right|+\left|C_{y}\right| \leq \frac{n+22}{3} \text { and }\left|A_{y}\right|+\left|B_{y}\right| \leq \frac{n+22}{3}
$$


Summing these three inequalities and dividing by 2 , we obtain that

$$
\left|A_{y}\right|+\left|B_{y}\right|+\left|C_{y}\right| \leq \frac{n+22}{2} .
$$

Furthermore, by the codegree condition,

$$
\frac{2 n-4}{3} \leq d(x, y) \leq\left|A_{y}\right|+\left|B_{y}\right|+\left|C_{y}\right|+\left(n-\left|V^{\prime}\right|\right) \leq \frac{n+22}{2}+4,
$$

where we apply $\left|V^{\prime}\right| \geq n-4$ from Lemma 2.4. Rearranging terms yields $\frac{n}{6} \leq \frac{49}{3}$, which contradicts our assumption $n>98$.

Set $V_{1}=\{y \in V \backslash\{x\}: \Gamma(x, y) \cap A=\emptyset\}, V_{2}=\{y \in V \backslash\{x\}: \Gamma(x, y) \cap B=\emptyset\}$ and $V_{3}=\{y \in V \backslash\{x\}: \Gamma(x, y) \cap C=\emptyset\}$. Without loss of generality, assume that

$$
\left|V_{1}\right| \leq\left|V_{2}\right| \leq\left|V_{3}\right|
$$

Claim 2.7 shows that $V_{1} \cup V_{2} \cup V_{3}$ covers $V(G) \backslash\{x\}$. We now show that in fact $V_{1}, V_{2}, V_{3}$ are pairwise disjoint, and $A \subseteq V_{1}, B \subseteq V_{2}$, and $C \subseteq V_{3}$. Suppose instead, that there exists $y \in V_{1} \cap V_{2}$. Then $\Gamma(x, y) \cap(A \cup B)=\emptyset$. By the codegree condition and Lemma 2.4.

$$
\frac{2 n-4}{3} \leq d(x, y) \leq\left|C_{y}\right|+\left(n-\left|V^{\prime}\right|\right) \leq \frac{n-2}{3}+4,
$$

which implies that $n \leq 14$, a contradiction.

Furthermore, consider $a^{\prime} \in A$. By Claim 2.6, $a^{\prime} x v \in E(G)$ for all but at most 8 vertices $v \in B \cup C$. By Lemma 2.4,

$$
|B|-8 \geq \frac{n-11}{3}-8>0
$$

which is strictly positive as $n>35$. Thus we have that $\Gamma\left(a^{\prime}, x\right)$ has a non-empty intersection with $B$; similarly we have that $\Gamma\left(a^{\prime}, x\right) \cap C \neq \emptyset$, from which we can finally deduce by Claim 2.7 that $\Gamma\left(a^{\prime}, x\right) \cap A=\emptyset$ and that $A \subseteq V_{1}$. Similarly we have $B \subseteq V_{2}$ and $C \subseteq V_{3}$.

Let $c^{\prime} \in C$. By the definition of $V_{3}$, we have $\Gamma\left(c^{\prime}, x\right) \subseteq V_{1} \cup V_{2}$. By the codegree assumption, it follows that

$$
\frac{2 n-4}{3} \leq d\left(c^{\prime}, x\right) \leq\left|V_{1}\right|+\left|V_{2}\right|=n-1-\left|V_{3}\right|,
$$

from which we get that $\left|V_{3}\right| \leq(n+1) / 3$. Since $n=3 m+2$, by (3) , we derive that $\left|V_{3}\right|=(n+1) / 3=$ $m+1$ and $\left|V_{1}\right| \leq\left|V_{2}\right| \leq(n+1) / 3$.

Claim 2.8. Let $y \in V_{i}$. Then $\Gamma(y, x)$ contains all but at most 6 vertices from $\bigcup_{j \neq i} V_{j}$ and no vertex from $V_{i}$.

Proof. Suppose without loss of generality that $y \in V_{1}$. Then by Claim 2.7, $A \cap \Gamma(y, x)=\emptyset$. Thus

$$
\frac{2 n-4}{3} \leq d(x, y) \leq\left|\Gamma(x, y) \cap\left(V_{2} \cup V_{3}\right)\right|+\left|\Gamma(x, y) \cap\left(V_{1} \backslash A\right)\right| \leq\left|\Gamma(x, y) \cap\left(V_{2} \cup V_{3}\right)\right|+4
$$


because $\left|V_{1} \backslash A\right| \leq n-\left|V^{\prime}\right| \leq 4$ by Lemma 2.4. Hence $\left|\Gamma(x, y) \cap\left(V_{2} \cup V_{3}\right)\right| \geq(2 n-16) / 3$. Since $\left|V_{i}\right| \leq(n+1) / 3$ for all $i$,

$$
\left|\left(V_{2} \cup V_{3}\right) \backslash \Gamma(x, y)\right| \leq 2 \frac{n+1}{3}-\frac{2 n-16}{3}=6 .
$$

This establishes the first part of our claim.

For the second part of our claim (namely, $\Gamma(y, x) \cap V_{1}=\emptyset$ ), suppose that $y y^{\prime} x \in E(G)$ for some $y^{\prime} \in V_{1}$. Then $\Gamma\left(y, y^{\prime}\right) \cap \Gamma(y, x) \cap \Gamma\left(y^{\prime}, x\right)=\emptyset$. Consequently,

$$
\frac{2 n-4}{3} \leq d\left(y, y^{\prime}\right) \leq 1+\left|V_{1}\right|-2+\left|\left(V_{2} \cup V_{3}\right) \backslash\left(\Gamma(y, x) \cap \Gamma\left(y^{\prime}, x\right)\right)\right| \leq 1+\frac{n+1}{3}-2+2 \cdot 6
$$

where in the last inequality we apply $\left|V_{1}\right| \leq(n+1) / 3$ and the first part of the claim. This implies that $n \leq 38$, a contradiction.

Claim 2.8 implies that $\Gamma\left(v_{3}, x\right) \subseteq V_{1} \cup V_{2}$ for all $v_{3} \in V_{3}$. Then $d\left(v_{3}, v\right)$ satisfies (4) with two inequalities replaced by equalities. Consequently all triples of the form $x v v_{3}$ with $v_{3} \in V_{3}$ and $v \in V_{1} \cup V_{2}$ are in $E(G)$

Claim 2.8 also implies that most $v_{1} \in V_{1}$ and $v_{2} \in V_{2}$ satisfy $x v_{1} v_{2} \in E(G)$. Fix such $v_{1}$ and $v_{2}$. Then $v_{1} v_{2} v_{3} \notin E(G)$ for any $v_{3} \in V_{3}$ otherwise $x v_{1} v_{2} v_{3}$ induces a copy of $K_{4}$. We thus have

$$
2 m \leq d\left(v_{1}, v_{2}\right) \leq\left|V_{1}\right|+\left|V_{2}\right|-1=2 m-1,
$$

a contradiction. This completes the proof of Theorem 1.1 in the case $n=3 m+2$.

\subsection{Other extremal constructions and stability}

Recall the construction $F_{1}(n)$ described in Section 2.1. There are other extremal families of 3-graphs for $K_{4}$-covering that are not isomorphic to subgraphs of $F_{1}(n)$.

Case 1: $n=3 m$. We partition $[n] \backslash\{x\}$ into three parts $V_{1}, V_{2}$ and $V_{3}$ with sizes $\left|V_{1}\right|=m-1$ and $\left|V_{2}\right|=\left|V_{3}\right|=m$. A collection $\mathcal{E}$ of pairs of vertices from different parts of $[n] \backslash\{x\}$ is called admissible if (i) every vertex $v_{1} \in V_{1}$ is contained in at most two pairs from $\mathcal{E}$, and (ii) every vertex $v \in V_{2} \sqcup V_{3}$ is contained in at most one pair from $\mathcal{E}$. Now let $F_{1}(\mathcal{E}, 3 m)$ be the 3-graph obtained from $F_{1}$ by deleting all triples $x u v$ and adding all tripartite triples $u v w$ (namely, $w \in V \backslash\{x\}$ is from the part different from the ones containing $u$ or $v$ ) for all $u v \in \mathcal{E}$. It is easy to see that $F_{1}(\mathcal{E}, 3 m)$ contains no $K_{4}$ covering $x$ and $\delta_{2}\left(F_{1}(\mathcal{E}, 3 m)\right)=\delta_{2}\left(F_{1}(3 m)\right)=2 m-2$.

Case 2: $n=3 m+1$. We partition $[n] \backslash\{x\}$ into three parts $V_{1}, V_{2}$ and $V_{3}$ with sizes $\left|V_{1}\right|=\left|V_{2}\right|=$ $\left|V_{3}\right|=m$. A collection $\mathcal{E}$ of pairs of vertices from different parts of $[n] \backslash\{x\}$ is called admissible if every vertex is contained in at most one pair from $\mathcal{E}$. Now let $F_{1}(\mathcal{E}, 3 m+1)$ be the 3 -graph obtained from $F_{1}$ by deleting all triples $x u v$ and adding all tripartite triples $u v w$ for all $u v \in \mathcal{E}$. It is easy ti see that $F_{1}(\mathcal{E}, 3 m+1)$ contains no $K_{4}$ covering $x$ and $\delta_{2}\left(F_{1}(\mathcal{E}, 3 m+1)\right)=\delta_{2}\left(F_{1}(3 m+1)\right)=2 m-1$. Case 3: $n=3 m+2$. We partition $[n] \backslash\{x\}$ into three parts $V_{1}, V_{2}$ and $V_{3}$ with sizes $\left|V_{1}\right|=\left|V_{2}\right|=m$ and $\left|V_{3}\right|=m+1$. A collection $\mathcal{E}$ of pairs of vertices from different parts of $[n] \backslash\{x\}$ is called admissible if (i) every vertex $v \in V_{1} \sqcup V_{2}$ is contained in at most 2 pairs from $\mathcal{E}$ and (ii) every vertex $v_{3} \in V_{3}$ is contained in at most 1 pair from $\mathcal{E}$. Now let $F_{1}(\mathcal{E}, 3 m+2)$ be the 3 -graph obtained from $F_{1}$ by deleting all triples $x u v$ and adding all tripartite triples $u v w$ for all $u v \in \mathcal{E}$. It is easy to see that $F_{1}(\mathcal{E}, 3 m+2)$ contains no $K_{4}$ covering $x$ and $\delta_{2}\left(F_{1}(\mathcal{E}, 3 m+2)\right)=\delta_{2}\left(F_{1}(3 m+2)\right)=2 m-1$. 
There is yet another extremal construction. Partition $[n] \backslash\{x\}$ into three parts $V_{1}, V_{2}$ and $V_{3}$ with sizes $\left|V_{1}\right|=m-1$ and $\left|V_{2}\right|=\left|V_{3}\right|=m+1$. In this context, a collection $\mathcal{E}$ of pairs of vertices from different parts of $[n] \backslash\{x\}$ is called admissible if (i) every vertex $v_{1} \in V_{1}$ is contained in at most 3 pairs from $\mathcal{E}$ and (ii) every vertex $v \in V_{2} \sqcup V_{3}$ is contained in at most 1 pair from $\mathcal{E}$. Let $F_{1}^{\prime}$ be the 3-graph on $[n]$ consisting of all triples $x u v$, where $u, v$ come from different parts, and all triples of $[n] \backslash\{x\}$ that are not tripartite. Now let $F_{1}^{\prime}(\mathcal{E}, 3 m+2)$ be the 3 -graph obtained from $F_{1}^{\prime}$ by deleting all triples $x u v$ and adding all tripartite triples $u v w$ for all $u v \in \mathcal{E}$. It is easy to see that $F_{1}^{\prime}(\mathcal{E}, 3 m+2)$ contains no $K_{4}$ covering $x$ and $\delta_{2}\left(F_{1}^{\prime}(\mathcal{E}, 3 m+2)\right)=\delta_{2}\left(F_{1}(3 m+2)\right)=2 m-1$.

We can show that the above constructions are all extremal configurations for $n$ sufficiently large $(n \geq 999)$. This can be done by first proving the following stability theorem.

Theorem 2.9 (Stability). Suppose $n \geq 4$ and $0<\delta \leq \frac{1}{429}$. Suppose that $G$ is a 3-graph on $n$ vertices with minimum codegree $\delta_{2}(G) \geq\left(\frac{2}{3}-\delta\right) n$ and that there is a vertex $x \in V(G)$ not contained in any copy of $K_{4}$ in $G$. Then there exists a tripartition $V_{1} \sqcup V_{2} \sqcup V_{3}$ of $V(G) \backslash\{x\}$ such that the following holds for all $i \in[3]$ and $j \neq i$ :

(i) there is no triple in $G$ of the form $x V_{i} V_{i}$;

(ii) all but at most $9 \delta n^{2}$ triples of the form $x V_{i} V_{j}$ are in $G$;

(iii) there are at most $4 \delta n^{3}$ triples in $G$ of the form $V_{1} V_{2} V_{3}$;

(iv) all but at most $6 \delta n^{3}$ triples of the form $V_{i} V_{i} V_{j}$ are in $G$;

(v) ||$V_{i}\left|-\frac{n-1}{3}\right| \leq 2 \delta n$.

Theorem 2.10. $\quad$ For $n \equiv 0 \bmod 3$ with $n \geq 858$, the extremal configurations for $c_{2}\left(n, K_{4}\right)$ are isomorphic to a subgraph of $F_{1}(\mathcal{E}, n)$ for some admissible $\mathcal{E}$.

- For $n \equiv 1 \bmod 3$ with $n \geq 715$, the extremal configurations for $c_{2}\left(n, K_{4}\right)$ are isomorphic to a subgraph of $F_{1}(\mathcal{E}, n)$ for some admissible $\mathcal{E}$.

- For $n \equiv 2 \bmod 3$ with $n \geq 1001$, the extremal configurations for $c_{2}\left(n, K_{4}\right)$ are isomorphic to a subgraph of $F_{1}(\mathcal{E}, n)$ or to a subgraph of $F_{1}^{\prime}(\mathcal{E}, n)$ for some admissible $\mathcal{E}$.

The proof of Theorem 2.9 is very similar to that of the case $n=3 m+2$ of Theorem 1.1, while the proof of Theorem 2.10 is a straightforward application of parts (i) and (ii) of Theorem 2.9. We therefore defer these proofs to the appendix.

\section{Covering thresholds for other 3-graphs}

\section{$3.1 K_{4}^{-}$}

Proof of the lower bound in Theorem 1.2. We construct a 3-graph $F_{2}(n)$ on $V=[n]$. Select a special vertex $x$. Split the remainder of the vertices into six parts $\sqcup_{i=1}^{6} V_{i}=V \backslash\{x\}$ with sizes as equal as possible, as follows:

$$
\left|V_{1}\right|-1 \leq\left|V_{6}\right| \leq\left|V_{5}\right| \leq\left|V_{4}\right| \leq\left|V_{3}\right| \leq\left|V_{2}\right| \leq\left|V_{1}\right|
$$


Put as the link of $x$ the blow-up of a 6 -cycle through the six parts, i.e. add all triples of the form $x V_{i} V_{i+1}$ for $i \in[6]$, winding round modulo 6 as necessary (identifying $V_{7}$ with $V_{1}$, and so on). Finally add those triples not involving $x$ which are not of type $V_{i} V_{i} V_{i+1}, V_{i} V_{i+1} V_{i+1}$ or $V_{i} V_{i+1} V_{i+2}$ for $i \in[6]$ (winding round modulo 6) to form the 3-graph $F_{2}(n)$.

Observe that the link graph of $x$ in $F_{2}(n)$ is triangle-free (being the blow-up of a 6 -cycle). Thus a putative $K_{4}^{-}$containing $x$ would have to be induced by a 4 -set $\{a, b, c, x\}$, with $a b c, a b x$ and $a c x$ all being triples of $F_{2}(n)$. Since $a b$ is in the link graph of $x$, we must have that $a, b$ come from different but adjacent parts $V_{i}, V_{i+1}$; by symmetry of $F_{2}(n)$, we may assume without loss of generality that $a \in V_{1}$ and $b \in V_{2}$. Since $a c x \in E\left(F_{2}(n)\right)$, it follows that $c \in V_{2}$ or $c \in V_{6}$. But by construction of $F_{2}(n)$, there are no triples of type $V_{6} V_{1} V_{2}$ or $V_{1} V_{2} V_{2}$, so that we cannot have in $a b c \in E\left(F_{2}(n)\right)$. Thus there is no copy of $K_{4}^{-}$in $F_{2}(n)$ covering $x$.

Let us now compute the minimum codegree of $F_{2}(n)$. Consider vertices $a_{i}, a_{i}^{\prime} \in V_{i}, a_{i+1} \in V_{i+1}$, $a_{i+2} \in V_{i+2}$ and $a_{i+3} \in V_{i+3}$. We have that $d\left(a_{i}, a_{i}^{\prime}\right)=n-3-\left|V_{i-1}\right|-\left|V_{i+1}\right|, d\left(a_{i}, a_{i+2}\right)=$ $n-3-\left|V_{i+1}\right|, d\left(a_{i}, a_{i+3}\right)=n-3$, and, lastly,

$$
d\left(a_{i}, x\right)=\left|V_{i-1}\right|+\left|V_{i+1}\right| \quad \text { and } \quad d\left(a_{i}, a_{i+1}\right)=1+\left|V_{i+3}\right|+\left|V_{i+4}\right| .
$$

Up to the choice of $i$, this covers all possible pairs in $F_{2}(n)$. The first three quantities are at least $n-3-2\left\lceil\frac{n-1}{6}\right\rceil \geq \frac{2 n}{3}-\frac{13}{3}$, which for $n \geq 12$ is greater than $\left\lfloor\frac{n-1}{3}\right\rfloor$. The last two quantities are both of order $\frac{n}{3}+O(1)$, however, and we analyse them more closely. Set $n=6 m+r$ for some $r \in\{0,1,2,3,4,5\}$. Then

$$
d\left(a_{i}, x\right) \geq \min _{i}\left(\left|V_{i-1}\right|+\left|V_{i+1}\right|\right)=\left|V_{6}\right|+\left|V_{4}\right|= \begin{cases}2 m-1 & \text { if } r=0 \\ 2 m & \text { if } 0<r<5 \\ 2 m+1 & \text { if } r=5\end{cases}
$$

and

$$
d\left(a_{i}, a_{i+1}\right) \geq \min _{i}\left(1+\left|V_{i+3}\right|+\left|V_{i+4}\right|\right)=1+\left|V_{5}\right|+\left|V_{6}\right|= \begin{cases}2 m & \text { if } r=0 \\ 2 m+1 & \text { if } 0<r \leq 5\end{cases}
$$

Thus

$$
c_{2}\left(n, K_{4}^{-}\right) \geq \delta_{2}\left(F_{2}(n)\right)= \begin{cases}2 m-1 & \text { if } r=0 \\ 2 m & \text { if } 0<r<5 \\ 2 m+1 & \text { if } r=5\end{cases}
$$

Proof of the upper bound in Theorem 1.2. Let $G$ be a 3-graph on $n \geq 4$ vertices. Suppose $\delta_{2}(G)>$ $\frac{n}{3}$. Pick an arbitrary vertex $x \in V(G)$. Let $a b x$ be any 3-edge containing $x$. We have $d(a, b)+$ $d(a, x)+d(b, x)-3>n-3$. So by the pigeonhole principle, there exists $c \in V(G) \backslash\{a, b, x\}$ which makes a 3-edge of $G$ with at least two of $a b, a x, b x$. The 4-set $a b c x$ then contains a copy of $K_{4}^{-}$in $G$ covering $x$, as required. This shows that $c_{2}\left(n, K_{4}^{-}\right) \leq\left\lfloor\frac{n}{3}\right\rfloor$.

Remark 3.1. Again we actually proved something stronger here: our argument establishes that for $\delta_{2}(G)$ above $\left\lfloor\frac{n}{3}\right\rfloor$, every triple of $E(G)$ can be extended to a copy of $K_{4}^{-}$.

Matching the upper and lower bounds obtained above, we obtain the set of possible values for $c_{2}\left(n, K_{4}^{-}\right)$claimed in Theorem 1.2 


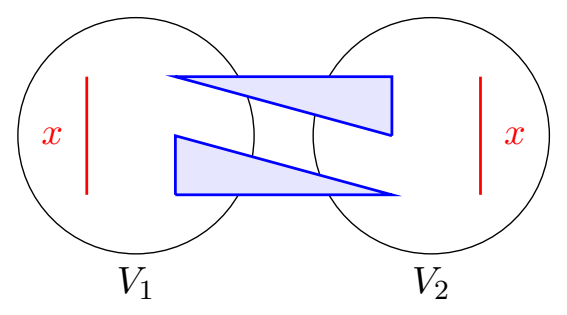

Figure 2: The 3-graph $F_{3}(n)$. The red pairs and the blue triples make up the link graph of $x$ and the remainder of $E\left(F_{3}\right)$ respectively.

Remark 3.2. We believe the gap between the upper and lower bounds for $c_{2}\left(n, K_{4}^{-}\right)$could be closed using similar (but more involved) stability arguments to those we used on to determine $c_{2}\left(n, K_{4}\right)$. However since such arguments would be non-trivial (the conjectured extremal configurations in this case are 6-partite) and would greatly increase the length of this paper, we do not pursue them here and leave open the determination of $c_{2}\left(n, K_{4}^{-}\right)$in the case where $n \equiv 0,3,4 \bmod 6$.

\section{$3.2 \quad C_{5}$}

Proof of the lower bound in Theorem 1.3. We construct a 3-graph $F_{3}(n)$ on $V=[n]$. Select a special vertex $x$. Split the remainder of the vertices into two parts $V \backslash\{x\}=V_{1} \sqcup V_{2}$ with sizes as equal as possible, $\left|V_{2}\right|-1 \leq\left|V_{1}\right| \leq\left|V_{2}\right|$. Form the link graph of $x$ by adding in all pairs internal to one of the parts, i.e. all pairs of the form $x V_{1} V_{1}$ or $x V_{2} V_{2}$. Next, add in all triples not containing $x$ and meeting both of the parts, i.e. all pairs of the form $V_{1} V_{1} V_{2}$ or $V_{1} V_{2} V_{2}$. This yields a 3-graph $F_{3}(n)$ with minimum codegree $\delta_{2}\left(F_{3}(n)\right)=\left|V_{1}\right|-1=\left\lfloor\frac{n-3}{2}\right\rfloor$, attained by $x$ and any vertex $a \in V_{1}$; see Figure 2.

Now there is no copy of $C_{5}$ covering $x \in F_{3}(n)$. Indeed, let $S=\left\{a_{1}, a_{2}, b_{1}, b_{2}\right\}$ be a set of four distinct vertices in $V \backslash\{x\}$ such that all of $a_{1} a_{2} x, a_{1} b_{1} x$ and $b_{1} b_{2} x$ are triples of $F_{3}(n)$. Then by construction these four vertices must all lie within the same part of $F_{3}(n)$. But by construction again we have that $S$ spans no triple of $F_{3}(n)$, whence $S \cup\{x\}$ does not contain a copy of $C_{5}$.

Proof of the upper bound in Theorem 1.3. Let $G=(V, E)$ be a 3 -graph on $n$ vertices with minimum codegree $\delta_{2}(G)>\frac{n}{2}$. Let $x$ be any vertex. Fix an edge $a b$ in the link graph $G_{x}$. Since $\delta_{1}\left(G_{x}\right)>n / 2$, $a$ and $b$ each has at least $\frac{n}{2}-1$ neighbours in $V \backslash\{x, u, v\}$. Hence $a$ and $b$ have a common neighbour $c$ in $G_{x}$. We shall use the triangle $\{a, b, c\}$ to find a copy of $C_{5}$ covering $x$. For this purpose, it is convenient to introduce the following notation. Given a 4-set of vertices $\left\{y_{1}, y_{2}, z_{1}, z_{2}\right\}$ from $V \backslash\{x\}$, write $y_{1} y_{1} \mid z_{1} z_{2}$ as a shorthand for the statement that all of $y_{1} z_{1} x, y_{1} y_{2} x, y_{2} z_{2} x, y_{2} z_{1} z_{2}$ and $y_{1} z_{1} z_{2}$ are in $E(G)$ (and in particular that $\left\{x, y_{1}, z_{1}, z_{2}, y_{2}\right\}$ contains a copy of $C_{5}$ covering $x$ ).

Lemma 3.3. There is either a copy of $C_{5}$ or a copy of $K_{4}$ covering $x$ in $G$.

Proof. If $a b c \in E(G)$ then the claim is immediate since $S=\{a, b, c, x\}$ induces a complete 3-graph. Assume therefore that $a b c \notin E(G)$. By our codegree assumption,

$$
d(a, b)+d(a, c)+d(b, c)+d(a, x)+d(b, x)+d(c, x)-9 \geq 6 \delta_{2}(G)-9>3(n-4) .
$$


Thus there exists $y \in V \backslash S$ which makes a 3-edge with at least four of the pairs $a b, a c, b c, a x$, $b x, c x$. It is now easy to check that $S \cup\{y\}$ contains either a $K_{4}$ or a $C_{5}$ covering $x$. Indeed by symmetry we may reduce the case-checking to the following three possibilities:

- if $y$ makes a 3-edge with $a b, b c, a c$ and $a x$, then $a b \mid c y$;

- if $y$ makes a 3-edge with $a b, a c$ and at least one of $b x$ or $c x$, then $b c \mid a y$;

- if $y$ makes a 3-edge with $a b$ and with both of $a x$ and $b x$, then $\{a, b, x, y\}$ induces a copy of $K_{4}$.

With a view towards proving Theorem 1.3. we may thus assume that there is a copy of $K_{4}$ covering $x$. Let $S=\{a, b, c, x\}$ be a 4 -set of vertices inducing such a $K_{4}$. By the codegree assumption,

$$
d(a, b)+d(a, c)+d(b, c)+d(a, x)+d(b, x)+d(c, x)-12 \geq 6 \delta_{2}(G)-12>3(n-4) .
$$

Thus there exists $y \in V \backslash S$ which makes a 3-edge with at least four of the pairs $a b, a c, b c, a x, b x$, $c x$. It is now easy to check that $S \cup\{y\}$ contains a copy of $C_{5}$ covering $x$. Indeed by symmetry we may reduce the case-checking to the following three possibilities:

- if $y$ makes a 3-edge with $a b, a c, b c$ and $a x$, then $a b \mid c y$;

- if $y$ makes a 3-edge with $a b, a c$ and at least one of $b x$ and $c x$, then $b c \mid a y$;

- if $y$ makes a 3-edge with $a b$ and with all of $a x, b x$ and $c x$, then $c y \mid a b$.

In all three cases we cover $x$ with a copy of $C_{5}$. The claimed upper bound on $c_{2}\left(n, C_{5}\right)$ follows.

Remark 3.4. Interestingly, as pointed out to us by Jie Han and Allan Lo, another very different construction attains the lower bound in Theorem [1.3. Take a balanced bipartition of [n] into two sets $V_{1}$ and $V_{2}$, with $\left|V_{1}\right| \leq\left|V_{2}\right|$. Now take all triples meeting $V_{1}$ in an even number of vertices to form a 3-graph $F_{4}(n)$. Note that $\delta_{2}\left(F_{4}(n)\right)=\min \left(\left|V_{1}\right|-1,\left|V_{2}\right|-2\right)$ (attained by pairs from $A \times B$ and $B^{(2)}$ respectively), which is exactly equal to $\left\lfloor\frac{n-3}{2}\right\rfloor$. Now, it is an easy exercise to check that every vertex $x \in V_{1}$ fails to be covered by a $C_{5}$, giving us a second proof that $c_{2}\left(n, C_{5}\right) \geq\left\lfloor\frac{n-3}{2}\right\rfloor$. In particular, we do not have stability for this problem: we have two near-extremal constructions which are easily seen to lie at edit distance $\Omega\left(n^{3}\right)$ from each other. Also we have that just below the codegree threshold for covering by $C_{5}$, we could have as many as $\left\lfloor\frac{n}{2}\right\rfloor$ uncovered vertices. This stands in sharp contrast with the situation for $K_{4}$ (see the discussion in Section 4 ).

\section{$3.3 \quad K_{5}^{-}$}

Proof of Theorem 1.4. For the lower bound, note that

$$
c_{2}\left(n, K_{5}^{-}\right) \geq c_{2}\left(n, K_{4}\right) \geq \delta_{2}\left(F_{1}(n)\right)=\left\lfloor\frac{2 n-5}{3}\right\rfloor .
$$

For the upper bound, let $G$ be a 3-graph on $n$ vertices with $\delta_{2}(G)>\frac{2 n-2}{3}$. By Theorem 1.1, for any vertex $x \in V(G)$ there is a triple $a_{1}, a_{2}, a_{3}$ such that $S=\left\{x, a_{1}, a_{2}, a_{3}\right\}$ induces a copy of $K_{4}$ in $G$. Now

$$
d\left(x, a_{1}\right)+d\left(x, a_{2}\right)+d\left(x, a_{3}\right)+d\left(a_{1}, a_{2}\right)+d\left(a_{1}, a_{3}\right)+d\left(a_{2}, a_{3}\right)-12>4(n-4),
$$


whence there exist $a_{4} \in V \backslash S$ which makes a 3-edge with at least 5 of the pairs from $S^{(2)}$. Thus $S \cup\left\{a_{4}\right\}$ contains a copy of $K_{5}^{-}$covering $x$. This shows that $c_{2}\left(n, K_{5}^{-}\right) \leq\left\lfloor\frac{2 n-2}{3}\right\rfloor$.

\subsection{The Fano plane}

Proposition 3.5. $\left\lfloor\frac{n}{2}\right\rfloor \leq c_{2}(n$, Fano $) \leq\left\lfloor\frac{2 n}{3}\right\rfloor$.

Proof. The lower bound is from the codegree threshold of the Fano plane: consider a bipartition of $[n]$ into two sets $V_{1} \sqcup V_{2}$ with $\left|V_{1}\right|=\left\lfloor\frac{n}{2}\right\rfloor$ and $\left|V_{2}\right|=\left\lceil\frac{n}{2}\right\rceil$, and adding all triples meeting both parts. The resulting 3-graph is easily seen to be Fano-free (it is 2-colourable, whereas the Fano plane is not) and has codegree $\left\lfloor\frac{n}{2}\right\rfloor$. For the upper bound, apply Lemma 2.1 with $F=$ Fano and $r=3$.

\section{$3.5 \quad F_{3,2}$}

Theorem 3.6. $1 / 3 \leq c_{2}\left(F_{3,2}\right) \leq 3 / 7$.

Proof. The lower bound is from the codegree density of $F_{3,2}$. An $F_{3,2}$-free construction on $n$ vertices with codegree $\left\lfloor\frac{n}{3}\right\rfloor-1$ is obtained by considering a tripartition of $[n]$ into three parts with sizes as equal as possible, $\left|V_{3}\right|-1 \leq\left|V_{1}\right| \leq\left|V_{2}\right| \leq\left|V_{3}\right|$ and adding all triples of the form $V_{i} V_{i} V_{i+1}$ (this is not actually best possible - see [7] for a determination of the precise codegree threshold and the extremal constructions attaining it).

For the upper bound, let $G$ be a 3-graph on $n$ vertices with $\delta_{2}(G)=c n$. Suppose there exists $x \in V(G)$ such that there is no copy of $F_{3,2}$ in $G$ covering $x$. This means that for every vertex $v \in V \backslash\{x\}, \Gamma(x, v)$ is an independent (3-edge-free) set in $G$, and moreover that for every 4-set $\{a, b, c, d\} \subseteq V(G)$, at least one of the triples $\{x a b, x c d, a b c, a b d\}$ is not in $E(G)$. For convenience, we shall write $a b \mid c d e$ as a short-hand for the statement that $\{a b c, a b d, a b e, c d e\}$ all are 3-edges of $G$.

We use the following technical lemma to deduce $c \leq 3 / 7+o(1)$.

Lemma 3.7. If there exist sets $A, B \subseteq V$ such that

1. A is a subset of $\Gamma(x, y)$ of size cn for some $y \in V \backslash\{x\}$, and $B$ is a subset of $V \backslash(A+\cup\{x\})$ of size cn, and

2. $B$ is independent in $G$ and the link graph $G_{x}$,

then $c \leq 3 / 7+o(1)$.

Proof of Lemma 3.7. Let $C=V \backslash(A \cup B)$. We have $|C|=n(1-2 c)$. By our assumption, $A$ is independent in $G$. By the codegree assumption, at least $\left(\begin{array}{c}|A| \\ 2\end{array}\right)(c n-|C|)$ triples of $G$ have two vertices in $A$ and one vertex in $B$. Consequently, at most $\left(\begin{array}{c}|A| \\ 2\end{array}\right)|C|=\left(\begin{array}{c}c n \\ 2\end{array}\right)(1-2 c) n$ triples of the form $A A B$ are missing from $G$.

On the other hand, let $b, b^{\prime} \in B$. Since $B$ is independent in $G$ and $G_{x}$, we have $\Gamma\left(b, b^{\prime}\right) \subseteq A \cup C$ and $\Gamma(b, x) \subseteq A \cup C$. Consequently $\left|\Gamma\left(b^{\prime}, x\right) \cap A\right| \geq c n-|C| \geq(3 c-1) n$ and

$$
\left|\Gamma\left(b, b^{\prime}\right) \cap \Gamma(b, x) \cap A\right| \geq 2(c n-|C|)-|A| \geq(5 c-2) n .
$$

For any $a \in \Gamma\left(b, b^{\prime}\right) \cap \Gamma(b, x) \cap A$ and any $a^{\prime} \in \Gamma\left(b^{\prime}, x\right) \cap A$, the triple $a a^{\prime} b$ must be absent from $G$ - otherwise $a b \mid a^{\prime} b x$. There are at least $\left(\begin{array}{c}(3 c-1) n \\ 2\end{array}\right)-\left(\begin{array}{c}(1-2 c) n \\ 2\end{array}\right)$ such pairs $\left(a, a^{\prime}\right)$ because, in general, 
there are at least $\left(\begin{array}{c}\left|A_{1}\right| \\ 2\end{array}\right)-\left(\begin{array}{c}\left|A_{1}\right|-\left|A_{2}\right| \\ 2\end{array}\right)$ pairs $\left(a_{1}, a_{2}\right)$ with $a \in A_{1}$ and $a_{2} \in A_{2}$ for arbitrary sets $A_{1}, A_{2}$ satisfying $\left|A_{1}\right| \geq\left|A_{2}\right|$.

There are thus at least $\left(\begin{array}{c}(3 c-1) n \\ 2\end{array}\right)-\left(\begin{array}{c}(1-2 c) n \\ 2\end{array}\right)$ distinct pairs $\left(a, a^{\prime}\right)$ for which $a a^{\prime} b \notin E(G)$. Summing over all $b \in B$, this gives us a total of at least $\left(\left(\begin{array}{c}(3 c-1) n \\ 2\end{array}\right)-\left(\begin{array}{c}(1-2 c) n \\ 2\end{array}\right)\right) c n A A B$ triples missing from $E(G)$. Combining this together with our upper bound on the number of missing $A A B$ triples yields the inequality

$$
\left(\left(\begin{array}{c}
(3 c-1) n \\
2
\end{array}\right)-\left(\begin{array}{c}
(1-2 c) n \\
2
\end{array}\right)\right) c n \leq\left(\begin{array}{c}
c n \\
2
\end{array}\right)(n(1-2 c)-1)
$$

which implies that

$$
\left((3 c-1)^{2}-(1-2 c)^{2}\right) \frac{n^{2}}{2} c n \leq \frac{c^{2} n^{2}}{2}(1-2 c) n+O\left(n^{2}\right) .
$$

This inequality in turn gives $c \leq 3 / 7+o\left(n^{-1}\right)$.

We now show that we can find $A, B \subseteq V$ satisfying the properties in Lemma 3.7 .

Suppose first of all that $G_{x}$ is not triangle-free. Let $y a_{1} a_{2}$ vertices spanning a triangle in $G_{x}$. Let $A$ be a subset of $\Gamma(x, y)$ of size $c n$. Then $A$ must be an independent set in $G$. Let $B$ be a subset of $\Gamma\left(a_{1}, a_{2}\right)$ in $V \backslash\{x\}$ of size $c n$. Then $B$ is disjoint from $A$ and is an independent set in $G_{x}$ - indeed if $b_{1} b_{2} \in G_{x}$ for some $b_{1}, b_{2} \in B$ then $a_{1} a_{2} \mid x b_{1} b_{2}$, a contradiction. We now show that $B$ is an independent set in $G$. Indeed, for every $b \in B, \Gamma(b, x)$ is a subset of $V \backslash B$ of size at least $c n$. Consider an arbitrary triple $\left\{b_{1}, b_{2}, b_{3}\right\}$ of distinct vertices from $B$. Since

$$
d\left(b_{1}, x\right)+d\left(b_{2}, x\right)+d\left(b_{3}, x\right)-2(n-|B|) \geq(3 c-2(1-c)) n=(5 c-2) n>0,
$$

by the pigeon-hole principle there exists $a \in A \cup C$ with $x a b_{1}, x a b_{2}, x a b_{3}$ all in $E(G)$. In particular $b_{1} b_{2} b_{3} \notin E(G)$, as otherwise we would have $a x \mid b_{1} b_{2} b_{3}$. It follows that $B$ must be an independent set in $G$. Thus $A, B$ satisfy the two properties in Lemma 3.7, and thus $c \leq 3 / 7+o(1)$

On the other hand, suppose $G_{x}$ was triangle-free. Let $y \in V \backslash\{x\}$, and let $A$ be a subset of $\Gamma(x, y)$ of size $c n$. Since $G_{x}$ is triangle-free and $x$ is not covered by an $F_{3,2}$-subgraph, $A$ forms an independent set in both $G_{x}$ and $G$. Let $a \in A$ be arbitrary, and let $B$ be a subset of $\Gamma(a, x)$ of size $c n$. Then $B$ is disjoint from $A$ and independent in $G_{x}$ (since $G_{x}$ is triangle-free). Thus $A, B$ satisfy the two properties in Lemma 3.7, and $c \leq 3 / 7+o(1)$. Thus $c_{2}\left(F_{3,2}\right) \leq 3 / 7$ as claimed.

\section{6 $K_{t}, t \geq 5$}

Proposition 3.8. For all $t \geq 5, c_{2}\left(K_{t}\right) \leq 1-1 /\left(\begin{array}{c}t-1 \\ 2\end{array}\right)$.

Proof. Applying Lemma 2.1 with $F=K_{t}$ and $r=\left(\begin{array}{c}t-1 \\ 2\end{array}\right)$, we get

$$
c_{2}\left(n, K_{t}\right) \leq\left\lfloor\left(1-\frac{1}{\left(\begin{array}{c}
t-1 \\
2
\end{array}\right)}\right) n-\frac{2 t-6}{t-2}\right\rfloor .
$$

We now derive a lower bound for the covering codegree density of $K_{t}$ by using (small) lowerbound constructions for the codegree threshold of $K_{t-1}$

Proposition 3.9. Suppose there exists a $K_{t-1}$-free 3-graph $H$ on $[m]$ with minimum codegree $\delta$. Then $c_{2}\left(K_{t}\right) \geq(\delta+2) / m$. 
Proof. We build a 3 -graph $G$ on $n=N m+1$ vertices as follows. Set $V=[n]$ and set aside a special vertex $x$. Partition $V \backslash\{x\}$ into $m$ sets $V_{1}, \ldots, V_{m}$, each of size $N$. Set as the link graph of $x$ all pairs of vertices from distinct parts. For every triple $i j k \in E(H)$, add to $G$ all 3-edges of the form $V_{i} V_{j} V_{k}$. Finally, add all triples of $V \backslash\{x\}$ that contain at least two vertices from one part. The minimum codegree of $G$ is

$$
\delta_{2}(G)=(\delta+2) N-1 \geq \frac{\delta+2}{m} n-2 .
$$

Now consider a $(t-1)$-set $S \subset V \backslash\{x\}$ that induce a $t$-clique in the link graph of $x$. By construction, these vertices must come from $t$ different parts of $V \backslash\{x\}$. Since $H$ is $K_{t-1}$-free, by our construction, some triple of $S$ is absent from $G$. Thus $S \cup\{x\}$ does not induce a copy of $K_{t}$ in $G$. Taking the limit as $n \rightarrow \infty$, the result follows.

Corollary 3.10. Let $t \geq 4$.

1. $c_{2}\left(K_{t}\right) \geq \frac{t-2}{t-1}$, and

2. $c_{2}\left(K_{t}\right) \geq \frac{2 t-6}{2 t-5}$ if $t \equiv 0,1 \bmod 3$.

Proof. For Part 1, we apply Proposition 3.9 with $H=K_{t-1}^{-}$(thus $m=t-1$ and $\delta=t-4$ ) and obtain $c_{2}\left(K_{t}\right) \geq \frac{t-2}{t-1}$.

For Part 2 , since $t \equiv 0,1 \bmod 3$, we have $2 t-5 \equiv 1,3 \bmod 6$, whence there exists a Steiner triple system $\mathcal{S}$ on the vertex set [2t-5]. It is easy to see that every set $T \subset[2 t-5]$ of $t-1$ vertices spans at least one triple from $\mathcal{S}$. Indeed, fix a vertex $a \in T$ : all the pairs of $T$ containing $a$ must have distinct neighbours under $\mathcal{S}$ in $[2 t-5] \backslash T$. Since $t-2>(2 t-5)-(t-1)$, this is impossible. Therefore the complement 3-graph $\overline{\mathcal{S}}$ is $K_{t-1}$-free. Applying Proposition 3.9 with $H=\overline{\mathcal{S}}$ (thus $m=2 t-5$ and $\delta=2 t-8)$, we obtain that $c_{2}\left(K_{t}\right) \geq \frac{2 t-6}{2 t-5}$.

Remark 3.11. 1. Combining Proposition 3.8 and Corollary 3.10 gives that $\frac{3}{4} \leq c_{2}\left(K_{5}\right) \leq \frac{5}{6}$ and $\frac{6}{7} \leq c_{2}\left(K_{6}\right) \leq \frac{9}{10}$.

2. Theorem 1.1 shows that the lower bound in Proposition 3.10 is tight in the case $t=4$. The bound is also tight in the trivial case $t=3$, since $c_{2}\left(n, K_{3}\right)=1=o(n)$. If this bound is tight in general, then we do not have stability for the covering codegree-threshold problem: while the 3 -edge $K_{3}$ and the Fano plane are the unique (up to isomorphism) Steiner triple systems on 3 and 7 vertices respectively, there are for example 11,084,874,829 non-isomorphic Steiner triple systems on 19 vertices (see [1, Section 4.5]).

\section{Concluding remarks}

There are many questions arising from our work. To begin with, we may ask which of the fundamental properties of Turán density and codegree density does the covering codegree density $c_{2}$ share. Explicitly:

1. do we have supersaturation? That is, if $\delta_{2}(G) \geq c_{2}(n, F)+\varepsilon n$ for some fixed $\varepsilon>0$, is it the case that every vertex in $G$ is contained in $\Omega\left(n^{|V(F)|-1}\right)$ copies of $F$ ? 
2. do we have blow-up invariance? Given a 3-graph $F$, we define the blow-up $F(t)$ to be the 3-graph on $V(F) \times[t]$ with 3-edges $\{(u, i)(v, j)(w, k): u v w \in E(F), i, j, k \in[t]\}$. Is it the case that for every $F$ and every fixed $t$ we have $c_{2}(F)=c_{2}(F(t))$ ?

3 . is the set of covering codegree densities $\left\{c_{2}(F): F\right.$ a 3-graph $\}$ dense in $[0,1]$, or does it have jumps?

The first two of these questions are addressed in a forthcoming work of the authors. In addition there are some natural variants of the covering codegree threshold $c_{2}(n, F)$ which may be interesting. What if instead of covering every vertex by a copy of $F$ we wanted to cover every pair? What if we wanted instead to be able to extend every 3-edge to a copy of $F$ ? It is not immediately clear whether the corresponding codegree-extremal functions behave similarly to $c_{2}(n, F)$ or not.

In a different direction, what if we asked for the threshold for covering all but at most $k$ vertices, for some $k \geq 1$ ? On the one hand, in the case of $C_{5}$ we observed in Remark 3.4 that this does not affect the value of the covering threshold very much. On the other hand, Theorem 2.9 implies that the threshold for covering all but at most 1 vertex with a copy of $K_{4}$ is at most $(2 / 3-c) n$ for some $c>0$ (therefore the problem is genuinely different from $c_{2}\left(n, K_{4}\right)$ ). Let us sketch a proof. Let $G$ be a 3-graph with $\delta_{2}(G) \geq(2 / 3-c) n$ for some $c>0$ sufficiently small. Suppose that $x \in V(G)$ is not covered by any copy of $K_{4}$. By Theorem [2.9, there is a partition $V_{1} \sqcup V_{2} \sqcup V_{3}$ of $V(G) \backslash\{x\}$ satisfying (i)-(v). If another vertex $y$ is not covered by any copy of $K_{4}$, then there is a partition $V_{1}^{\prime} \sqcup V_{2}^{\prime} \sqcup V_{3}^{\prime}$ of $V(G) \backslash\{y\}$ satisfying (i)-(v) as well. Because of (iii) and (iv), these two partitions essentially coincide. Now consider $\Gamma(x, y)$, which has size at least $(2 / 3-c) n$. There are about $(2 / 3-c)(1 / 3-3 c) n^{2} / 2$ pairs $u, v \in \Gamma(x, y)$ coming from different parts of $V_{1} \cap V_{1}^{\prime}, V_{2} \cap V_{2}^{\prime}, V_{3} \cap V_{3}^{\prime}$. Since $(2 / 3-c)(1 / 3-3 c) n^{2} / 2>2 \cdot 10 c n^{2}$ (for $c$ sufficiently small), by (ii), there exists a pair $u, v \in \Gamma(x, y)$ such that both $u v x$ and $u v y$ are edges of $G$. This implies that $\{u, v, x, y\}$ spans a copy of $K_{4}$, a contradiction. The authors note that the bound on $c$ given by this argument can be significantly improved; this is the subject of future work.

Finally, it would be interesting to determine the value of $c_{2}(F)$ when $F$ is the Fano plane or $F_{3,2}$, and to have if not a tight result then at least a reasonable guess as to the value of $c_{2}\left(K_{t}\right)$ for $t \geq 5$. An investigation of $c_{1}(n, F)$ when $F=K_{4}^{-}$and $F=K_{4}$ would also be desirable.

We should note here that for such small 3-graphs $F$ the problem of proving upper bounds for $c_{1}$ or $c_{2}$ should be amenable to flag algebra computations by following the approach of [7] to encode the minimum degree/codegree constraint. Note however that one will need to do computation with non-uniform hypergraphs, containing a mixture of 2-edges (from the link graph of an uncovered vertex $x$ ) and 3-edges.

\section{Acknowledgements}

The authors thank Jie Han and Allan Lo for pointing out the alternative lower bound construction for Theorem 1.3. The first author is also grateful for the hospitality of Georgia State University during a research visit which led to the present paper.

\section{References}

[1] C.J. Colbourn and J.H. Dinitz. Handbook of combinatorial designs. CRC press, 2006. 
[2] A. Czygrinow. Tight co-degree condition for packing of loose cycles in 3-graphs. Journal of Graph Theory, in press.

[3] A. Czygrinow, L. DeBiasio, and B. Nagle. Tiling 3-uniform hypergraphs with $K_{4}^{3}-2 e$. Journal of Graph Theory, 75(2):124-136, 2014.

[4] A. Czygrinow and B. Nagle. A note on codegree problems for hypergraphs. Bull. Inst. Combin. Appl, 32:63-69, 2001.

[5] L. DeBiasio and T. Jiang. On the co-degree threshold for the Fano plane. European Journal of Combinatorics, 36:151-158, 2014.

[6] V. Falgas-Ravry. On the codegree density of complete 3-graphs and related problems. Electronic Journal of Combinatorics, 20(4):P28, 2013.

[7] V. Falgas-Ravry, E. Marchant, O. Pikhurko, and E.R. Vaughan. The codegree threshold for 3-graphs with independent neighbourhoods. SIAM J. Discrete Math., 29(3):1504-1539, 2015.

[8] V. Falgas-Ravry, O. Pikhurko, and E.R. Vaughan. The codegree density of $K_{4}^{-}$. In preparation, 2015.

[9] P. Frankl and Z. Füredi. An exact result for 3-graphs. Discrete mathematics, 50:323-328, 1984.

[10] Z. Füredi. Turán type problems. In Surveys in combinatorics, volume 166. Cambridge Univ. Press Cambridge, 1991.

[11] W. Gao and J. Han. Minimum codegree threshold for $C_{6}^{3}$-factors in 3-uniform hypergraphs. arXiv preprint arXiv:1508.05152, 2015.

[12] A. Hajnal and E. Szemerédi. Proof of a conjecture of Erdös. Combinatorial theory and its applications, 2:601-623, 1970.

[13] J. Han, Allan Lo, A. Treglown, and Y. Zhao. Exact minimum codegree threshold for $K_{4}^{-}$factors. arXiv preprint arXiv:1509.02577, 2015.

[14] J. Han, C. Zang, and Y. Zhao. Minimum vertex degree thresholds for tiling complete 3-partite 3-graphs. arXiv preprint arXiv:1503.08730, 2015.

[15] P. Keevash. A hypergraph regularity method for generalized Turán problems. Random Structures \& Algorithms, 34(1):123-164, 2009.

[16] P. Keevash. Hypergraph Turán Problems. Surveys in combinatorics, 2011.

[17] P. Keevash and R. Mycroft. A geometric theory for hypergraph matching. Memoirs of the American Mathematical Society, 233(1098), 2015.

[18] P. Keevash and Y. Zhao. Codegree problems for projective geometries. Journal of Combinatorial Theory, Series B, 97(6):919-928, 2007.

[19] P. Kirkman. On a problem in combinatorics. Cambridge Dublin Math. J., 2:191-204, 1847.

[20] D. Kühn and D. Osthus. Loose Hamilton cycles in 3-uniform hypergraphs of high minimum degree. Journal of Combinatorial Theory, Series B, 96(6):767-821, 2006.

[21] D. Kühn and D. Osthus. The minimum degree threshold for perfect graph packings. Combinatorica, 29(1):65-107, 2009.

[22] A. Lo and K. Markström. Minimum codegree threshold for $K_{4}^{-}$factors. Journal of Combinatorial Theory, Series A, 120(3):708-721, 2013.

[23] A. Lo and K. Markström. F-factors in hypergraphs via absorption. Graphs and Combinatorics, 31(3):679-712, 2015.

[24] E. Marchant. Graphs with weighted colours and hypergraphs. PhD thesis, University of Cambridge, 2011.

[25] D. Mubayi. The co-degree density of the Fano plane. Journal of Combinatorial Theory, Series 
B, 95(2):333-337, 2005.

[26] D. Mubayi and V. Rödl. On the Turán number of triple systems. Journal of Combinatorial Theory, Series A, 100(1):136-152, 2002.

[27] D. Mubayi and Y. Zhao. Co-degree density of hypergraphs. Journal of Combinatorial Theory, Series A, 114(6):1118-1132, 2007.

[28] R. Mycroft. Packing $k$-partite $k$-uniform hypergraphs. to appear in Journal of Combinatorial Theory, Series A, 2014.

[29] B. Nagle. Turán-Related Problems for Hypergraphs. Congressus numerantium, pages 119-128, 1999.

[30] V. Rödl and A. Ruciński. Dirac-type questions for hypergraphs — a survey (or more problems for Endre to solve). In An Irregular Mind, pages 561-590. Springer, 2010.

[31] V. Rödl, A. Ruciński, and E. Szemerédi. Perfect matchings in large uniform hypergraphs with large minimum collective degree. Journal of Combinatorial Theory, Series A, 116(3):613-636, 2009.

[32] P. Turán. On an extremal problem in graph theory. Mat. Fiz. Lapok, 48:436-452, 1941.

[33] Y Zhao. Recent advances on Dirac-type problems for hypergraphs. arXiv preprint arXiv:1508.06170, 2015.

\section{Appendix: proof of Theorem 2.9 and Theorem 2.10}

Proof of Theorem [2.9. We run through the proof of the case $n=3 m+2$ of Theorem 2.9, replacing the codegree assumption $\delta_{2}(G)=2 m-2$ by the assumption $\delta_{2}(G) \geq \frac{2 n}{3}-\delta n$. This gives us new versions of our claims and lemmas with error terms involving $\delta n$, and conditions on $n$ being sufficiently large replaced by conditions on $\delta$ being sufficiently small. For the sake of completeness, we derive them below.

Let $G$ be a 3 -graph on $n$ vertices with $\delta_{2}(G) \geq\left(\frac{2}{3}-\delta\right) n$, for some $\delta$ : $0<\delta \leq 1 / 429$. Suppose there is a vertex $x$ of $G$ not contained in any copy of $K_{4}$. As $2 / 3-\delta>1 / 2$, the link graph $G_{x}$ contains a triangle $\{a b, b c, a c\}$. Set $S=\{a, b, c, x\}$ and for each $y \in V \backslash S$, define $S_{y}$ as in Lemma 2.3. By Lemma 2.3. $S_{y}$ is a subset of $S^{1, c}, S^{1, b}, S^{1, a}, S^{2, a}, S^{2, b}, S^{2, c}$ or $S^{3}$. For $i \in\{1,2\}$ and $j \in\{a, b, c\}$, write $s_{i, j}$ for the number of vertices $y \in V \backslash S$ for which $S_{y}=S^{i, j}$, and write $s_{i}$ for the sum $s_{i, a}+s_{i, b}+s_{i, c}$. Finally let $s_{0}$ be the number of vertices $y \in V \backslash S$ such that $S_{y} \neq S^{i, j}$ for any $i \in\{1,2\}$ and $j \in\{a, b, c\}$. Note that $\left|S_{y}\right| \leq 3$ for such $y$. We know that $s_{1}+s_{2}+s_{0}=n-4$. Furthermore, by the codegree assumption,

$$
\begin{gathered}
3\left(\frac{2 n}{3}-\delta n\right) \leq d(a, x)+d(b, x)+d(c, x) \leq 2 s_{1}+s_{2}+3 s_{0}+6 \\
6\left(\frac{2 n}{3}-\delta n\right) \leq d(a, x)+d(b, x)+d(c, x)+d(a, b)+d(b, c)+d(c, a) \leq 4 s_{1}+4 s_{2}+3 s_{0}+9
\end{gathered}
$$

Substituting $s_{0}=n-4-s_{1}-s_{2}$ into (5) and (6) yields that $s_{1}+2 s_{2} \leq n+3 \delta n-6$ and $s_{1}+s_{2} \geq n-6 \delta n+3$, respectively. Combining the two inequalities we have just obtained, we get

$$
s_{2} \leq 9 \delta n-9 \quad \text { and } \quad s_{1} \geq n-15 \delta n+12 .
$$


We now show as before that the weight of $s_{1}$ splits almost equally between $s_{1, a}, s_{1, b}, s_{1, c}$. Note that

$$
\frac{2 n}{3}-\delta n \leq d(b, c) \leq n-3-s_{1, a}
$$

from which it follows that $s_{1, a} \leq \frac{n}{3}+\delta n-3$. Similarly we derive that $s_{1, b}, s_{1, c} \leq \frac{n}{3}+\delta n-3$. Consequently

$$
s_{1, a}=s_{1}-s_{1, b}-s_{1, c} \geq n-15 \delta n+12-2\left(\frac{n}{3}+\delta n-3\right)=\frac{n}{3}-17 \delta n+18 .
$$

Similarly $s_{1, b}$ and $s_{1, c}$ satisfy the same lower bound. Set $A=\left\{y \in V \backslash S: S_{y}=S^{1, a}\right\} \cup\{a\}$, $B=\left\{y \in V \backslash S: S_{y}=S^{1, b}\right\} \cup\{b\}$ and $C=\left\{y \in V \backslash S: S_{y}=S^{1, c}\right\} \cup\{c\}$. Set $V^{\prime}=A \cup B \cup C \cup\{x\}$. The calculations above have established the following lemma.

Lemma 6.1 (New version of Lemma 2.4).

$$
\left|V^{\prime}\right| \geq n-15 \delta n+16, \quad \text { and } \quad \frac{n}{3}-17 \delta n+19 \leq|A|,|B|,|C| \leq \frac{n}{3}+\delta n-2 .
$$

Let $\mathcal{B}$ be the collection of 3 -edges of $G$ of the form $x A A, x B B, x C C$ (the 'bad' triples). Let $\mathcal{M}$ be the collection of non-edges of $G$ of the form $x A B, x A C, x B C$ (the 'missing' triples). Viewing $\mathcal{B}$ and $\mathcal{M}$ as 3 -graphs on $V^{\prime}$, for two distinct vertices $v_{1}, v_{2} \in V^{\prime}$, we let $d_{\mathcal{B}}\left(v_{1}, v_{2}\right)$ denote their codegree in $\mathcal{B}$ and $d_{\mathcal{M}}\left(v_{1}, v_{2}\right)$ their codegree in $\mathcal{M}$.

Claim 6.2 (New version of Claim 2.5). For every $v \in V^{\prime} \backslash\{x\}, d_{\mathcal{B}}(v, x) \leq 18 \delta n-20$.

Proof. Suppose without loss of generality that $v \in A$. If $v=a$, then $d_{\mathcal{B}}(v, x)=0$ because $G$ contains no 3 -edges of the form $x a A$. We thus assume that $v \neq a$. The bad triples for the pair $(v, x)$ are triples of the form $a^{\prime} v x$ for $a^{\prime} \in A \backslash\{a, v\}$. Suppose $a^{\prime} v x \in \mathcal{B}$. Then since there is no $K_{4}$ in $G$ containing $x$, and since, by the definition of $A, a^{\prime} b x, v b x, a^{\prime} c x$ and $v c x$ are all in $G$, it must be the case that both of $a^{\prime} v b$ and $a^{\prime} v c$ are missing from $G$. Further if $c^{\prime} \in C \cap \Gamma(v, x)$ then all of $c^{\prime} v x, b v x, c^{\prime} b x$ are in $G$, whence $b c^{\prime} v$ is absent from $G$. Similarly for any $b^{\prime} \in B$, at most one of $b^{\prime} c v$, $b^{\prime} x v$ is in $G$. Finally since $b c v \notin E(G), b$ and $c$ are contained in exactly one of $\Gamma(b, v), \Gamma(c, v)$, and $\Gamma(x, v)$. To summarize, a vertex $y$ in $V^{\prime}$ can lie in at most two of $\Gamma(b, v), \Gamma(c, v)$ and $\Gamma(x, v)$ unless $y$ is in $\Gamma_{\mathcal{B}}(x, v)$ (and lies in exactly one of those joint neighbourhoods) or is in $\{b, c, v\}$ (and lies in at most one of those joint neighbourhoods). Together with our codegree assumption, this gives us

$$
\begin{aligned}
3\left(\frac{2 n}{3}-\delta n\right) & \leq d(b, v)+d(c, v)+d(x, v) \leq 2\left|V^{\prime}\right|-d_{\mathcal{B}}(v, x)-4+3\left(n-\left|V^{\prime}\right|\right) \\
& =3 n-\left|V^{\prime}\right|-4-d_{\mathcal{B}}(v, x) \leq 2 n+15 \delta n-20-d_{\mathcal{B}}(v, x),
\end{aligned}
$$

where we apply $\left|V^{\prime}\right| \geq n-15 \delta n+16$ from Lemma 6.1 in the last inequality. It follows that $d_{\mathcal{B}}(v, x) \leq 18 \delta n-20$, as claimed.

Claim 6.3 (New version of claim 2.6). For every $v \in V^{\prime} \backslash\{x\}, d_{\mathcal{M}}(v, x) \leq 36 \delta n-40$. 
Proof. Suppose without loss of generality that $v \in A$. Then by the codegree assumption, Claim 6.2 and the bound on $|A|$ from Lemma 6.1 we have

$$
\begin{aligned}
\frac{2 n}{3}-\delta n \leq d(v, x) & \leq n-1-|A|+d_{\mathcal{B}}(v, x)-d_{\mathcal{M}}(v, x) \\
& \leq n-1-\frac{n}{3}+17 \delta n-19+18 \delta n-20-d_{\mathcal{M}}(v, x),
\end{aligned}
$$

which gives that $d_{\mathcal{M}}(v, x) \leq 36 \delta n-40$ as claimed.

Claim 6.4 (New version of Claim 2.7). Provided $\delta \leq \frac{1}{429}$, for every $y \in V(G) \backslash\{x\}, \Gamma(y, x)$ has a non-empty intersection with at most two of the parts $A, B$ and $C$.

Proof. Let $y \in V(G) \backslash\{x\}$. Set $A_{y}=A \cap \Gamma(x, y), B_{y}=B \cap \Gamma(x, y)$ and $C_{y}=C \cap \Gamma(x, y)$. Suppose none of $A_{y}, B_{y}, C_{y}$ is empty. Fix $a^{\prime} \in A_{y}$. For $b^{\prime} \in B_{y}$, if $b^{\prime} \in \Gamma\left(a^{\prime}, x\right)$, then $a^{\prime} b^{\prime} y \notin E(G)-$ otherwise $\left\{a^{\prime}, b^{\prime}, x, y\right\}$ spans a copy of $K_{4}$. Similarly, for $c^{\prime} \in C_{y} \cap \Gamma\left(a^{\prime}, x\right)$, we have $a^{\prime} c^{\prime} y \notin E(G)$. Hence,

$$
\frac{2 n}{3}-\delta n \leq d\left(a^{\prime}, y\right) \leq n-2-\left|B_{y} \cap \Gamma\left(a^{\prime}, x\right)\right|-\left|C_{y} \cap \Gamma\left(a^{\prime}, x\right)\right| .
$$

Claim 6.3 gives that $d_{\mathcal{M}}\left(a^{\prime}, x\right) \leq 36 \delta n-40$. Consequently,

$$
\left|B_{y} \cap \Gamma\left(a^{\prime}, x\right)\right|+\left|C_{y} \cap \Gamma\left(a^{\prime}, x\right)\right|=\left|B_{y}\right|+\left|C_{y}\right|-d_{\mathcal{M}}\left(a^{\prime}, x\right) \geq\left|B_{y}\right|+\left|C_{y}\right|-36 \delta n+40
$$

This implies that

$$
\frac{2 n}{3}-\delta n \leq n-2-\left|B_{y}\right|-\left|C_{y}\right|+36 \delta n-40,
$$

which yields $\left|B_{y}\right|+\left|C_{y}\right| \leq \frac{n}{3}+37 \delta n-42$. Similarly by considering any vertex $b^{\prime} \in B_{y}$ and any vertex $c^{\prime} \in C_{y}$ we obtain that

$$
\left|A_{y}\right|+\left|C_{y}\right| \leq \frac{n}{3}+37 \delta n-42 \text { and }\left|A_{y}\right|+\left|B_{y}\right| \leq \frac{n}{3}+37 \delta n-42 .
$$

Summing these three inequalities and dividing by 2 , we obtain that

$$
\left|A_{y}\right|+\left|B_{y}\right|+\left|C_{y}\right| \leq \frac{n+111 \delta n-126}{2} .
$$

Furthermore, by the codegree condition,

$$
\frac{2 n}{3}-\delta n \leq d(x, y) \leq\left|A_{y}\right|+\left|B_{y}\right|+\left|C_{y}\right|+\left(n-\left|V^{\prime}\right|\right) \leq \frac{n+141 \delta n-158}{2},
$$

where we apply $\left|V^{\prime}\right| \geq n-15 \delta n+16$ from Lemma 6.1, Rearranging terms yields $\frac{(1-429 \delta) n}{6} \leq-\frac{158}{2}$, which is a contradiction as $\delta \leq 1 / 429$.

Set $V_{1}=\{y \in V \backslash\{x\}: \Gamma(x, y) \cap A=\emptyset\}, V_{2}=\{y \in V \backslash\{x\}: \Gamma(x, y) \cap B=\emptyset\}$ and $V_{3}=\{y \in V \backslash\{x\}: \Gamma(x, y) \cap C=\emptyset\}$. Without loss of generality, assume that $\left|V_{1}\right| \leq\left|V_{2}\right| \leq\left|V_{3}\right|$. Claim 6.4 shows that $V_{1} \cup V_{2} \cup V_{3}$ covers $V(G) \backslash\{x\}$. We now show that in fact $V_{1}, V_{2}, V_{3}$ are 
pairwise disjoint, and $A \subseteq V_{1}, B \subseteq V_{2}$, and $C \subseteq V_{3}$. Suppose instead that there exists $y \in V_{1} \cap V_{2}$. Then $\Gamma(x, y) \cap(A \cup B)=\emptyset$. By the codegree condition and Lemma 6.1 ,

$$
\frac{2 n}{3}-\delta n \leq d(x, y) \leq\left|C_{y}\right|+\left(n-\left|V^{\prime}\right|\right) \leq \frac{n}{3}+\delta n-2+15 \delta n-16=\frac{n}{3}+16 \delta n-18 .
$$

Rearranging terms yields $\frac{(1-51 \delta) n}{3} \leq-18$, which for $\delta \leq 1 / 51$ is a contradiction.

Furthermore, consider $a^{\prime} \in A$. By Claim 6.3, $a^{\prime} x v \in E(G)$ for all but at most $36 \delta n-40$ vertices $v \in B \cup C$. By Lemma 6.1,

$$
|B|-36 \delta+40 \geq \frac{(1-159 \delta) n}{3}+59
$$

which is strictly positive when $\delta \leq 1 / 159$. Thus we have that $\Gamma\left(a^{\prime}, x\right)$ has a non-empty intersection with $B$; similarly we have that $\Gamma\left(a^{\prime}, x\right) \cap C \neq \emptyset$, from which we can finally deduce by Claim 6.4 that $\Gamma\left(a^{\prime}, x\right) \cap A=\emptyset$ and that $A \subseteq V_{1}$. Similarly we have $B \subseteq V_{2}$ and $C \subseteq V_{3}$.

We claim that

$$
\forall i \in\{1,2,3\}, \quad\left|V_{i}\right| \leq n / 3+\delta n-1
$$

Indeed, let $c^{\prime} \in C$. By the definition of $V_{3}$, we have $\Gamma\left(c^{\prime}, x\right) \subseteq V_{1} \cup V_{2}$. By the codegree assumption, it follows that

$$
\frac{2 n}{3}-\delta n \leq d\left(c^{\prime}, x\right) \leq\left|V_{1}\right|+\left|V_{2}\right|=n-1-\left|V_{3}\right|,
$$

from which we get that $\left|V_{3}\right| \leq n / 3+\delta n-1$, as claimed. By (77), we have $\left|V_{i}\right|=n-1-\bigcup_{j \neq i}\left|V_{j}\right| \geq$ $n / 3-2 \delta n+1$ and consequently,

$$
\frac{4}{3}-2 \delta n \leq\left|V_{i}\right|-\frac{n-1}{3} \leq \delta n-\frac{2}{3} .
$$

This gives Part (v) of Theorem 2.9.

Claim 6.5 (New version of Claim 2.8). Let $y \in V_{i}$. Then provided $\delta \leq \frac{1}{429}, \Gamma(y, x)$ contains all but at most $18 \delta n-18$ vertices from $\bigcup_{j \neq i} V_{j}$ and no vertex from $V_{i}$.

Proof. Suppose without loss of generality that $y \in V_{1}$. Then by Claim 6.4, $A \cap \Gamma(y, x)=\emptyset$. Thus $\frac{2 n}{3}-\delta n \leq d(x, y) \leq\left|\Gamma(x, y) \cap\left(V_{2} \cup V_{3}\right)\right|+\left|\Gamma(x, y) \cap\left(V_{1} \backslash A\right)\right| \leq\left|\Gamma(x, y) \cap\left(V_{2} \cup V_{3}\right)\right|+15 \delta n-16$ since $\left|V_{1} \backslash A\right| \leq n-\left|V^{\prime}\right| \leq 15 \delta n-16$ by Lemma 6.1. Hence $\left|\Gamma(x, y) \cap\left(V_{2} \cup V_{3}\right)\right| \geq \frac{2 n}{3}-16 \delta n+16$. By (7),

$$
\left|\left(V_{2} \cup V_{3}\right) \backslash \Gamma(x, y)\right| \leq 2\left(\frac{n}{3}+\delta n-1\right)-\left(\frac{2 n}{3}-16 \delta n+16\right)=18 \delta n-18 .
$$

This establishes the first part of our claim.

For the second part of our claim (namely that $\Gamma(y, x) \cap V_{1}=\emptyset$ ), suppose that $y y^{\prime} x \in E(G)$ for some $y^{\prime} \in V_{1}$. Then $\Gamma\left(y, y^{\prime}\right) \cap \Gamma(y, x) \cap \Gamma\left(y^{\prime}, x\right)=\emptyset$. Consequently,

$$
\begin{aligned}
\frac{2 n}{3}-\delta n \leq d\left(y, y^{\prime}\right) & \leq 1+\left|V_{1}\right|-2+\left|\left(V_{2} \cup V_{3}\right) \backslash\left(\Gamma(y, x) \cap \Gamma\left(y^{\prime}, x\right)\right)\right| \\
& \leq 1+\frac{n}{3}+\delta n-1-2+2(18 \delta n-18)
\end{aligned}
$$

where in the last inequality we applied (7) and the first part of the claim. This implies that $\frac{(1-114 \delta) n}{3} \leq-38$, a contradiction when $\delta \leq 1 / 114$. 
This establishes Part (i) of Theorem [2.9, By Claim [6.5, the total number of missing $x V_{i} V_{j}$ edges, $i \neq j$ is at most

$$
\frac{1}{2} \sum_{i}(18 \delta n-18)\left|V_{i}\right|=\frac{1}{2}(n-1)(18 \delta n-18)<9 \delta n^{2},
$$

establishing Part (ii) of the theorem. Since a triple $v_{1} v_{2} v_{3}$ with $v_{i} \in V_{i}$ is an edge of $G$ only if one of the $x v_{i} v_{i+1}$ triples is missing, by Part (ii) and Equation (7), there can be at most $9 \delta n^{2}(n / 3+\delta n)<$ $4 \delta n^{3}$ such triples in total, establishing Part (iii) of the Theorem.

Finally we need to bound the number of non-edges of $G$ intersecting exactly two of $V_{1}, V_{2}, V_{3}$. Fix $v_{1} \in V_{1}$ and $v_{2} \in V_{2}$. Given a set $S \subseteq V(G)$, let $d\left(v_{1}, v_{2}, S\right)=\left|\Gamma\left(v_{1}, v_{2}\right) \cap S\right|$ denote the number of neighbours of $v_{1}$ and $v_{2}$ in $S$ and $\bar{d}\left(v_{1}, v_{2}, S\right)=\left|S \backslash \Gamma\left(v_{1}, v_{2}\right)\right|$. By the codegree condition, we have

$$
d\left(v_{1}, v_{2}, V_{1} \cup V_{2}\right) \geq \frac{2}{3} n-\delta n-1-d\left(v_{1}, v_{2}, V_{3}\right) .
$$

Together with (7), this implies that

$$
\begin{aligned}
\bar{d}\left(v_{1}, v_{2}, V_{1} \cup V_{2}\right) & \leq\left|V_{1}\right|+\left|V_{2}\right|-2-\left(\frac{2}{3} n-\delta n-1-d\left(v_{1}, v_{2}, V_{3}\right)\right) \\
& \leq 2\left(\frac{n}{3}+\delta n-1\right)-2-\frac{2}{3} n+\delta n+1+d\left(v_{1}, v_{2}, V_{3}\right) \\
& =3 \delta n-3+d\left(v_{1}, v_{2}, V_{3}\right),
\end{aligned}
$$

The number of non-edges of $G$ in the form of $V_{1} V_{1} V_{2}$ or $V_{1} V_{2} V_{2}$ is thus

$$
\frac{1}{2} \sum_{v_{1} \in V_{1}, v_{2} \in V_{2}} \bar{d}\left(v_{1}, v_{2}, V_{1} \cup V_{2}\right) \leq \frac{1}{2}\left(\left|V_{1}\right|\left|V_{2}\right|(3 \delta n-3)+e\left(V_{1}, V_{2}, V_{3}\right)\right)
$$

where $e\left(V_{1}, V_{2}, V_{3}\right)$ denotes the number of tripartite edges. We know that $e\left(V_{1}, V_{2}, V_{3}\right) \leq 9 \delta n^{2}(n / 3+$ $\delta n)$. Thus the number of non-edges of $G$ intersecting exactly two of $V_{1}, V_{2}, V_{3}$ is at most

$$
\begin{aligned}
\frac{1}{2} \sum_{i}\left|V_{i}\right|\left|V_{i+1}\right|(3 \delta n-3)+\frac{3}{2} e\left(V_{1}, V_{2}, V_{3}\right) & \leq \frac{n-1}{2}\left(\frac{n}{3}+\delta n-1\right)(3 \delta n-3)+\frac{9}{2} \delta n^{3}(1+3 \delta) \\
& <\frac{n}{2}\left(\frac{n}{3}+\delta n\right) 3 \delta n+\frac{9}{2} \delta n^{3}(1+3 \delta)<6 \delta n^{3},
\end{aligned}
$$

where we applied (77) in the second inequality. This establishes Part (iv) of the Theorem.

Proof of Theorem 2.10. Case 1: $n=3 m \geq 858$. Let $G$ be a 3-graph on $n=3 m$ vertices with $\delta_{2}(G)=2 m-2=\left(\frac{2}{3}-\frac{2}{n}\right) n$. Suppose $x \in V(G)$ is not covered by any copy of $K_{4}$. Since $\delta=\frac{2}{n} \leq \frac{1}{429}$, we can apply Theorem 2.9 to obtain a tripartition $V_{1} \sqcup V_{2} \sqcup V_{3}=V(G) \backslash\{x\}$ satisfying conditions (i)-(v) from Theorem 2.9. Assume without loss of generality that $\left|V_{1}\right| \leq\left|V_{2}\right| \leq\left|V_{3}\right|$. For any vertex $v_{3} \in V_{3}$, we have (by condition (i))

$$
2 m-2 \leq d\left(x, v_{3}\right) \leq\left|V_{1}\right|+\left|V_{2}\right|=3 m-1-\left|V_{3}\right|,
$$

from which it follows that $\left|V_{3}\right| \leq m+1$. 
Suppose $\left|V_{3}\right|=m+1$. The condition (i) tells us that all triples of the form $x v v_{3}$ with $v \in V_{1} \sqcup V_{2}$ and $v_{3} \in V_{3}$ must be in $G$, for otherwise $d\left(x, v_{3}\right)<2 m-2=\delta_{2}(G)$. Now consider any pair of vertices $v_{1} \in V_{1}, v_{2} \in V_{2}$ for which $x v_{1} v_{2}$ is in $G$ (such pairs must exist by condition (ii), say). For every $v_{3} \in V_{3}$, both of $x v_{1} v_{3}$ and $x v_{2} v_{3}$ are in $G$, whence the tripartite triple $v_{1} v_{2} v_{3}$ must be absent from $G$ (since otherwise $x v_{1} v_{2} v_{3}$ would induce a copy of $K_{4}$ in $G$ ). Thus the codegree of $v_{1}, v_{2}$ is at most $\left|V_{1}\right|+\left|V_{2}\right|-1=2 m-3$, a contradiction.

Thus $\left|V_{3}\right|=m$, whence $\left|V_{2}\right|=m$ also and $\left|V_{1}\right|=m-1$. Now, by condition (i), for every $v_{3} \in V_{3}$ at most one triple $v v_{3} x$ with $v \in V_{1} \sqcup V_{2}$ can be missing in $G$, as otherwise $d\left(v_{3}, x\right)<2 m-2$; similarly for every $v_{2} \in V_{2}$ at most one triple $v v_{2} x$ with $v \in V_{1} \sqcup V_{3}$ is missing, and for every $v_{1} \in V_{1}$ at most 2 triples $v v_{1} x$ with $v \in V_{2} \sqcup V_{3}$ are missing. Further a tripartite triple $v_{1} v_{2} v_{3}$ can be included in $G$ only if one of the triples $x v_{1} v_{2}, x v_{2} v_{3}, x v_{1} v_{3}$ is missing from $G$. This shows that $G$ must be (isomorphic to) a subgraph of $F_{1}(\mathcal{E}, 3 m)$ for some admissible collection of pairs $\mathcal{E}$.

Case 2: $n=3 m+1 \geq 715$. Let $G$ be a 3-graph on $n=3 m+1$ vertices with minimum codegree $\delta_{2}(G)=2 m-1=\left(\frac{2}{3}-\frac{5}{3 n}\right) n$. Suppose $x \in V(G)$ is not covered by any copy of $K_{4}$. Since $\delta=$ $\frac{5}{3 n} \leq \frac{1}{429}$, we can apply Theorem 2.9 to obtain a tripartition of $V(G) \backslash\{x\}=V_{1} \sqcup V_{2} \sqcup V_{3}$ satisfying conditions (i)-(v) from Theorem 2.9. Assume without loss of generality that $\left|V_{1}\right| \leq\left|V_{2}\right| \leq\left|V_{3}\right|$.

For any vertex $v_{3} \in V_{3}$, we have (by condition (i))

$$
2 m-1 \leq d\left(x, v_{3}\right) \leq\left|V_{1}\right|+\left|V_{2}\right|=3 m-\left|V_{3}\right|,
$$

from which it follows that $\left|V_{3}\right| \leq m+1$. If $\left|V_{3}\right|=m+1$, then by the codegree assumption and condition (i) all triples of the form $x v v_{3}$ with $v_{3} \in V_{3}$ and $v \in V_{1} \sqcup V_{2}$ are in $E(G)$. Now consider vertices $v_{1} \in V_{1}$ and $v_{2} \in V_{2}$ for which $x v_{1} v_{2} \in E(G)$ (which must exist by condition (ii), say). The tripartite triple $v_{1} v_{2} v_{3}$ does not lie in $E(G)$ for any $v_{3} \in V_{3}$, since otherwise $x v_{1} v_{2} v_{3}$ would induce a copy of $K_{4}$. Thus $d\left(v_{1}, v_{2}\right) \leq\left|V_{1}\right|+\left|V_{2}\right|-1=2 m-2<2 m-1$, a contradiction. We must thus have $\left|V_{1}\right|=\left|V_{2}\right|=\left|V_{3}\right|=m$.

Now by condition (i) and the codegree assumption, for every vertex $v_{i} \in V_{i}$ all but at most 1 of the triples $x v v_{i}$ with $v \in V(G) \backslash\left(V_{i} \cup\{x\}\right)$ must be in $E(G)$. Furthermore a tripartite triple $v_{1} v_{2} v_{3}$ can belong to $G$ only if one of the triples $x v_{1} v_{2}, x v_{2} v_{3}, x v_{3} v_{1}$ is absent from $G$. This shows that $G$ is (isomorphic to) a subgraph of $F_{1}(\mathcal{E}, 3 m+1)$ for some admissible collection of pairs $\mathcal{E}$.

Case 3: $n=3 m+2 \geq 1001$.

Let $G$ be a 3-graph on $n=3 m+2$ vertices with minimum codegree $\delta_{2}(G)=2 m-1=\left(\frac{2}{3}-\frac{7}{3 n}\right) n$. Suppose $x \in V(G)$ is not covered by any copy of $K_{4}$. Since $\delta=\frac{7}{3 n} \leq \frac{1}{429}$, we find a tripartition of $V(G) \backslash\{x\}=V_{1} \sqcup V_{2} \sqcup V_{3}$ satisfying conditions (i)-(v) from Theorem [2.9, Assume without loss of generality that $\left|V_{1}\right| \leq\left|V_{2}\right| \leq\left|V_{3}\right|$.

For any vertex $v_{3} \in V_{3}$, by condition (i), we have

$$
2 m-1 \leq d\left(x, v_{3}\right) \leq\left|V_{1}\right|+\left|V_{2}\right|=3 m+1-\left|V_{3}\right|,
$$

from which it follows that $\left|V_{3}\right| \leq m+2$. If $\left|V_{3}\right|=m+2$, then by the codegree assumption and condition (i) all triples of the form $x v v_{3}$ with $v_{3} \in V_{3}$ and $v \in V_{1} \sqcup V_{2}$ are in $E(G)$. Now consider vertices $v_{1} \in V_{1}$ and $v_{2} \in V_{2}$ for which $x v_{1} v_{2} \in E(G)$ (which must exist by condition (ii), say). The tripartite triple $v_{1} v_{2} v_{3}$ does not lie in $E(G)$ for any $v_{3} \in V_{3}$, since otherwise $x v_{1} v_{2} v_{3}$ would induce a copy of $K_{4}$. Thus $d\left(v_{1}, v_{2}\right) \leq\left|V_{1}\right|+\left|V_{2}\right|-1=2 m-2<2 m-1$, a contradiction. We must thus have $\left|V_{3}\right| \leq m+1$. Our assumption that $\left|V_{1}\right| \leq\left|V_{2}\right| \leq \mid V_{3}$ then implies that $\left|V_{3}\right| \leq m+1$ and that $\left|V_{2}\right| \in\{m, m+1\}$. We have two subcases to consider. 
Case 3a: $\left|V_{1}\right|=\left|V_{2}\right|=m$. Condition (i) and the codegree assumption together imply that for every $v \in V_{i}$, all but at most 2 of the triples of the form $x v\left(V \backslash\left(V_{i} \cup\{x\}\right)\right)$ must be in $E(G)$ if $i \in\{1,2\}$, and all but at most 1 if $i=3$. Further a tripartite triple $v_{1} v_{2} v_{3}$ can be in $E(G)$ only if one of $x v_{1} v_{2}, x v_{2} v_{3}, x v_{3} v_{1}$ is absent from $E(G)$. This shows that $G$ must be (isomorphic to) a subgraph of $F_{1}(\mathcal{E}, 3 m)$ for some admissible collection of pairs $\mathcal{E}$.

Case 3b: $\left|V_{1}\right|=m-1,\left|V_{2}\right|=m+1$. Condition (i) and the codegree assumption together imply that for every $v \in V_{i}$, all but at most 1 of the triples of the form $x v\left(V \backslash\left(V_{i} \cup\{x\}\right)\right)$ must be in $E(G)$ if $i \in\{2,3\}$, and all but at most 3 if $i=1$. Further a tripartite triple $v_{1} v_{2} v_{3}$ is in $E(G)$ only if one of $x v_{1} v_{2}, x v_{2} v_{3}, x v_{3} v_{1}$ is absent from $E(G)$. This shows that $G$ must be (isomorphic to) a subgraph of $F_{1}^{\prime}(\mathcal{E}, 3 m)$ for some admissible collection of pairs $\mathcal{E}$. 